\title{
Measurement of the Top Quark Pair Production Cross Section in Proton-Proton Collisions at $\sqrt{s}=13 \mathrm{TeV}$
}

\author{
V. Khachatryan et al. ${ }^{*}$ \\ (CMS Collaboration) \\ (Received 18 October 2015; published 5 February 2016)
}

\begin{abstract}
The top quark pair production cross section is measured for the first time in proton-proton collisions at $\sqrt{s}=13 \mathrm{TeV}$ by the CMS experiment at the CERN LHC, using data corresponding to an integrated luminosity of $43 \mathrm{pb}^{-1}$. The measurement is performed by analyzing events with at least one electron and one muon of opposite charge, and at least two jets. The measured cross section is $746 \pm 58$ (stat) \pm 53 (syst) \pm 36 (lumi) pb, in agreement with the expectation from the standard model.
\end{abstract}

DOI: 10.1103/PhysRevLett.116.052002

The measurement of $t \bar{t}$ production at a center-of-mass energy not previously accessed has great discovery potential for physics beyond the standard model (SM), because new phenomena can significantly enhance the $t \bar{t}$ cross section. The increased energy also allows for a test of the production mechanism, dominated at the CERN LHC by gluon-gluon fusion, and of the validity of the theory of quantum chromodynamics (QCD). Furthermore, top quark production is an important source of background in many searches for physics beyond the SM, and its accurate evaluation is important. Previously, large samples of top quark events were collected in proton-proton collisions at the LHC at $\sqrt{s}=7$ and $8 \mathrm{TeV}$ and used to study $t \bar{t}$ production in different final states by the ATLAS [1-11] and CMS [12-20] collaborations.

This Letter presents the first measurement of the $t \bar{t}$ production cross section $\sigma_{t \bar{t}}$ at $\sqrt{s}=13 \mathrm{TeV}$, utilizing data corresponding to an integrated luminosity of $43 \mathrm{pb}^{-1}$ recorded by the CMS experiment. In the SM, top quarks are produced predominantly in $t \bar{t}$ pairs via the strong interaction, and each top quark decays almost exclusively to a $W$ boson and a $b$ quark. For this study, we select events that contain at least one electron and one muon of opposite charge, and at least two jets.

The central feature of the CMS detector [21] is a superconducting solenoid of $6 \mathrm{~m}$ internal diameter, providing a magnetic field of $3.8 \mathrm{~T}$. A silicon pixel and strip tracker, a lead tungstate crystal electromagnetic calorimeter (ECAL), and a brass and scintillator hadron calorimeter, each composed of a barrel and two endcap sections, are located within the solenoid volume. Muons are measured in gas-ionization detectors embedded in the steel flux-return

*Full author list given at the end of the article.

Published by the American Physical Society under the terms of the Creative Commons Attribution 3.0 License. Further distribution of this work must maintain attribution to the author(s) and the published article's title, journal citation, and DOI. yoke outside the solenoid. A two-tier trigger system selects the most interesting $p p$ collisions for offline analysis. A more detailed description of the CMS detector, together with a definition of its coordinate system and kinematic variables, can be found in Ref. [21].

We use several Monte Carlo (MC) generator programs to simulate signal and background processes. The nextto-leading-order (NLO) POWHEG (v2) $[22,23]$ generator is used to generate $t \bar{t}$ signal events, assuming a top quark mass of $m_{t}=172.5 \mathrm{GeV}$ [24]. We utilize the NNPDF3.0 NLO [25] parton distribution functions (PDF) in the MC calculations. The events are interfaced to PYTHIA (v8.205) $[26,27]$ with the CUETP8M1 tune [28,29] to simulate parton showering, hadronization, and the underlying event. An alternative sample is obtained using the HERWIG++ (v2.7.1) [30] program to model the parton shower. Another sample of $t \bar{t}$ events is generated using MG5_AMC@NLO (v5_2.2.2) [31] and MADSPIN [32] generators, and again PYTHIA (v8.205) for parton showering, hadronization, and the underlying event. The MC generators have been validated by comparing to unfolded differential distributions of $t \bar{t}$ production at $\sqrt{s}=$ $8 \mathrm{TeV}$ [33].

Background events are simulated by the MG5_AMC@ NLO (v5_2.2.2) generator for $W+$ jets production and Drell-Yan (DY) quark-antiquark annihilation into leptonantilepton pairs through virtual photon or $\mathrm{Z}$ boson exchange, with normalization taken from data. Associated top quark and $W$ boson production $(t W)$ is simulated using POWHEG (v1) $[34,35]$ and PYTHIA (v8.205), and is normalized to the approximate next-to-next-toleading-order (NNLO) cross section [36]. The contributions from $W W, W Z$ and $Z Z$ (referred to as $V V$ ) processes are simulated with PYTHIA (v8.205), and normalized to their NLO cross sections [37]. All other backgrounds are estimated from control samples extracted from collision data. The simulated samples include additional interactions per bunch crossing (pileup). On average, about 20 collisions per bunch crossing are present in our data. 
The SM prediction for the $t \bar{t}$ production cross section at $\sqrt{s}=13 \mathrm{TeV}$ is calculated with the TOP++ program [38] at NNLO in perturbative QCD, including soft-gluon resummation at next-to-next-to-leading-log order (NNLL) [39-44], assuming $m_{t}=172.5 \mathrm{GeV}$. The result is $\sigma_{\bar{t} \overline{\mathrm{NLO}}+\mathrm{NNLL}}^{\mathrm{N}}=832_{-29}^{+20}(\mathrm{scale}) \pm 35\left(\mathrm{PDF}+\alpha_{s}\right) \mathrm{pb}$. The expected yields for signal in all figures and tables are normalized to this value. The first uncertainty reflects uncertainties in the factorization and renormalization scales, $\mu_{\mathrm{F}}$ and $\mu_{\mathrm{R}}$. The second uncertainty, associated with the PDFs and strong coupling constant $\alpha_{\mathrm{s}}$, is obtained by following the PDF4LHC prescription $[45,46]$ using the MSTW2008 68\% C.L. NNLO [47,48], CT10 NNLO [49,50], and NNPDF2.3 5f FFN [51] PDF sets.

At the trigger level, events are required to contain one electron and one muon, where the electron has transverse momentum $p_{\mathrm{T}}>12 \mathrm{GeV}$ and the muon has $p_{\mathrm{T}}>$ $17 \mathrm{GeV}$, or the electron has $p_{\mathrm{T}}>17 \mathrm{GeV}$ and the muon has $p_{\mathrm{T}}>8 \mathrm{GeV}$. Offline, particle candidates are reconstructed with the CMS particle-flow (PF) algorithm [52,53]. The PF algorithm reconstructs and identifies each individual particle using an optimized combination of information from the various elements of the CMS detector.

Events are selected to contain one electron [54] and one muon [55] of opposite charge, both of which are required to have $p_{\mathrm{T}}>20 \mathrm{GeV}$ and $|\eta|<2.4$ (but excluding electrons within a small region of $|\eta|$ between the barrel and endcap sections of the ECAL). The electron and muon candidates are required to be sufficiently isolated from nearby jet activity as follows. For each electron and muon candidate, a cone of $\Delta R=0.3$ and $\Delta R=0.4$, respectively, is constructed around the direction of the track at the event vertex, where $\Delta R$ is defined as $\sqrt{(\Delta \eta)^{2}+(\Delta \phi)^{2}}$, and $\Delta \eta$ and $\Delta \phi$ are the distances in pseudorapidity and azimuthal angle. Excluding the contribution from the lepton candidate, the scalar sum of the $p_{\mathrm{T}}$ of all particle candidates that are inside $\Delta R$ and are consistent with arising from the chosen primary event vertex is calculated to define a relative isolation discriminant, $I_{\text {rel }}$, through the ratio of this sum to the $p_{\mathrm{T}}$ of the lepton candidate. The neutral-particle contribution to $I_{\text {rel }}$ is corrected for pileup based on the average energy density deposited by neutral particles in the event. This corresponds to an average $p_{\mathrm{T}}$ from pileup determined event-by-event that is subtracted from the summed scalar $p_{\mathrm{T}}$ in the isolation cone. An electron and muon candidate is selected if they have respective values of $I_{\text {rel }}<0.11$ and $I_{\text {rel }}<0.12$.

In events with more than one pair of leptons passing the above selection, the two leptons of opposite charge and different flavor with the largest $p_{\mathrm{T}}$ are selected for further study. Events with $\tau$ leptons contribute to the measurement only if they decay to electrons or muons that satisfy the selection requirements, and are included in the $\mathrm{MC}$ simulations.
The efficiency of the lepton selection is measured using a "tag-and-probe" method in same-flavor dilepton events enriched in $\mathrm{Z}$ boson candidates, as described in Refs. [19,56]. Differences in the event topology with respect to $t \bar{t}$ production are accounted for as a systematic uncertainty. In the current data set, the measured values for the combined identification and isolation efficiencies are typically $92 \%$ for muons and $77 \%$ for electrons. Based on a comparison of lepton selection efficiencies in data and simulation, the event yield in simulation is corrected using $p_{\mathrm{T}^{-}}$and $\eta$-dependent data-to-simulation scale factors (SF) to provide consistency with data. They have average values of 1.00 for muons and 0.96 for electrons.

Candidate events with dilepton invariant masses of $m_{e \mu}<20 \mathrm{GeV}$ are removed to suppress backgrounds, mainly from low-mass DY processes. Jets are reconstructed from the PF particle candidates using the anti- $k_{\mathrm{T}}$ clustering algorithm [57] with a distance parameter of 0.4, optimized for the running conditions at higher center-of-mass energy. The jet energy is corrected for pileup in a manner similar to that used to find the energy within the lepton isolation cone. Jet energy corrections are also applied as a function of jet $p_{\mathrm{T}}$ and $\eta$ [58] to data and simulation. Events are required to have at least two reconstructed jets with $p_{\mathrm{T}}>30 \mathrm{GeV}$ and $|\eta|<2.4$.

Backgrounds in this analysis arise primarily from $t W$, DY, and $V V$ events in which at least two leptons are produced. Background yields from $t W$ and $V V$ events are estimated from simulation. The $e^{ \pm} \mu^{\mp}$ DY background normalization is estimated from data using the " $R_{\text {out } / \text { in }}$ " method $[19,59,60]$, where events with $e^{+} e^{-}$and $\mu^{+} \mu^{-}$final states are explored as follows. A data-to-simulation normalization factor is estimated from the number of events within the $\mathrm{Z}$ boson mass window in data, and extrapolated to the number of events outside the $\mathrm{Z}$ mass window with corrections based on control regions in data enriched in DY events. This factor is found to be $1.04 \pm 0.16$ (stat).

Other background sources, such as $t \bar{t}$ or $W+$ jets events with decays into one lepton and jets, can contaminate the signal sample if a jet is incorrectly reconstructed as a lepton, or an event contains a lepton from the decay of bottom or charm hadrons. These are grouped into the nonprompt-lepton category, together with contributions that can arise, for example, from the decays of mesons, photon conversions to $e^{+} e^{-}$pairs in the material of the detector, or effects from detector resolution. The nonprompt-lepton background is estimated from an extrapolation of a control region of same-sign (SS) dilepton events to the signal region of opposite-sign (OS) dileptons. The SS control region is defined using the same criteria as used for the nominal signal region, except requiring $e \mu$ pairs of the same charge. The SS dilepton events predominantly contain at least one misidentified lepton. Other SM processes, such as DY, $t W, V V$ and $t \bar{t}$ dilepton production have significantly smaller contributions, and are estimated using 

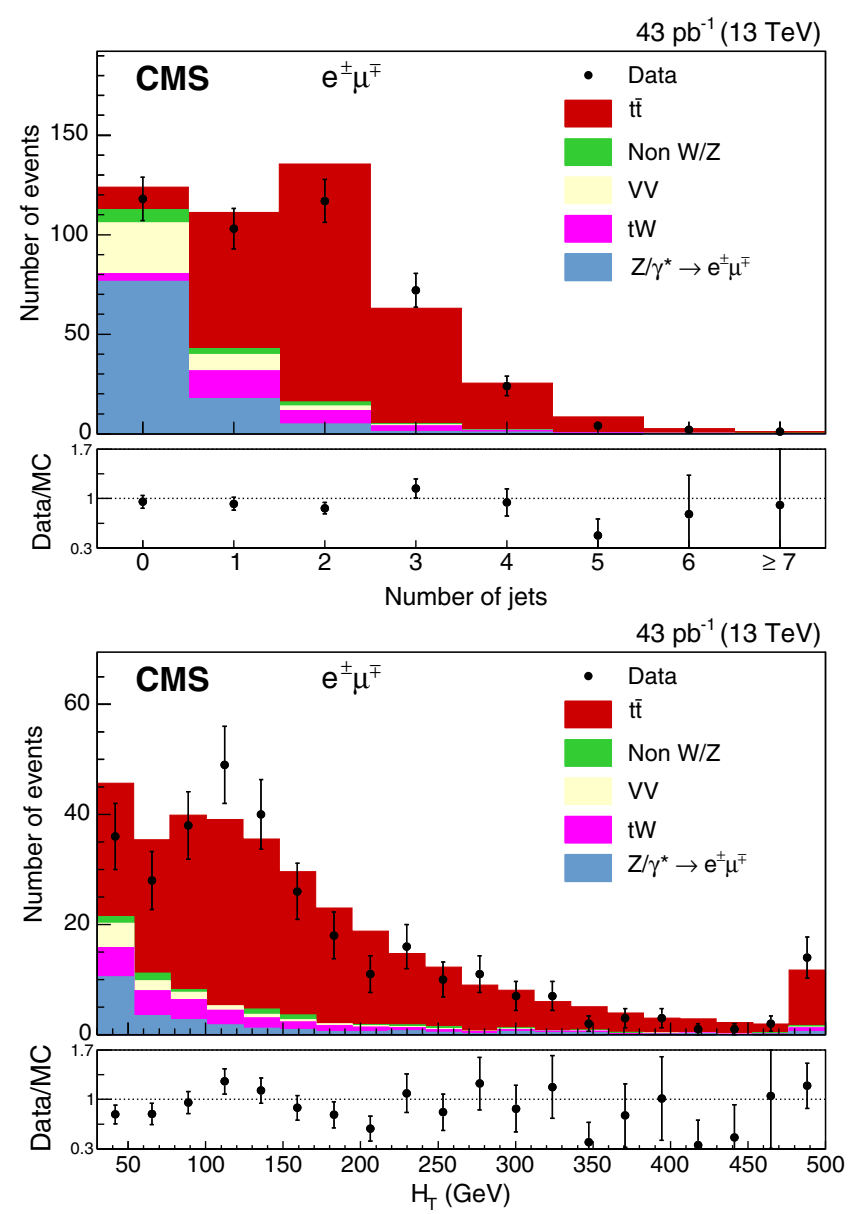

FIG. 1. The distributions in (top) the jet multiplicity, and (bottom) $H_{\mathrm{T}}$ in events passing the dilepton criteria. The expected distributions for $t \bar{t}$ signal and individual backgrounds are shown after implementing data-based corrections; the last bin contains the overflow events. The ratios of data to the sum of the expected yields are given at the bottom of each panel.

simulation. The scaling from the SS control region in data to the signal region is performed using an extrapolation factor, extracted from MC simulation, given by the ratio of the number of OS events with misidentified leptons to the number of SS events with misidentified leptons. From the eight same-sign events observed in data, the expected contamination of $1.7 \pm 0.4$ events due to DY, $t W, V V$ and $t \bar{t}$ dilepton production is subtracted, and the result is multiplied by the OS to SS ratio of $1.4 \pm 0.3$ to obtain an estimate of $8.5 \pm 4.4$ nonprompt lepton events contaminating the signal, including statistical and systematic uncertainties. This agrees with predictions from $\mathrm{MC}$ simulations of semileptonic $t \bar{t}$ and $W+$ jets events.

Figure 1 (top) shows the multiplicity of jets and (bottom) the scalar $p_{\mathrm{T}}$ sum of all jets $\left(H_{\mathrm{T}}\right)$ for events passing the dilepton criteria. Agreement is observed between data and the predictions for signal and background.

After requiring at least two jets, we obtain the plots presented in Fig. 2, where (top) shows the distribution in
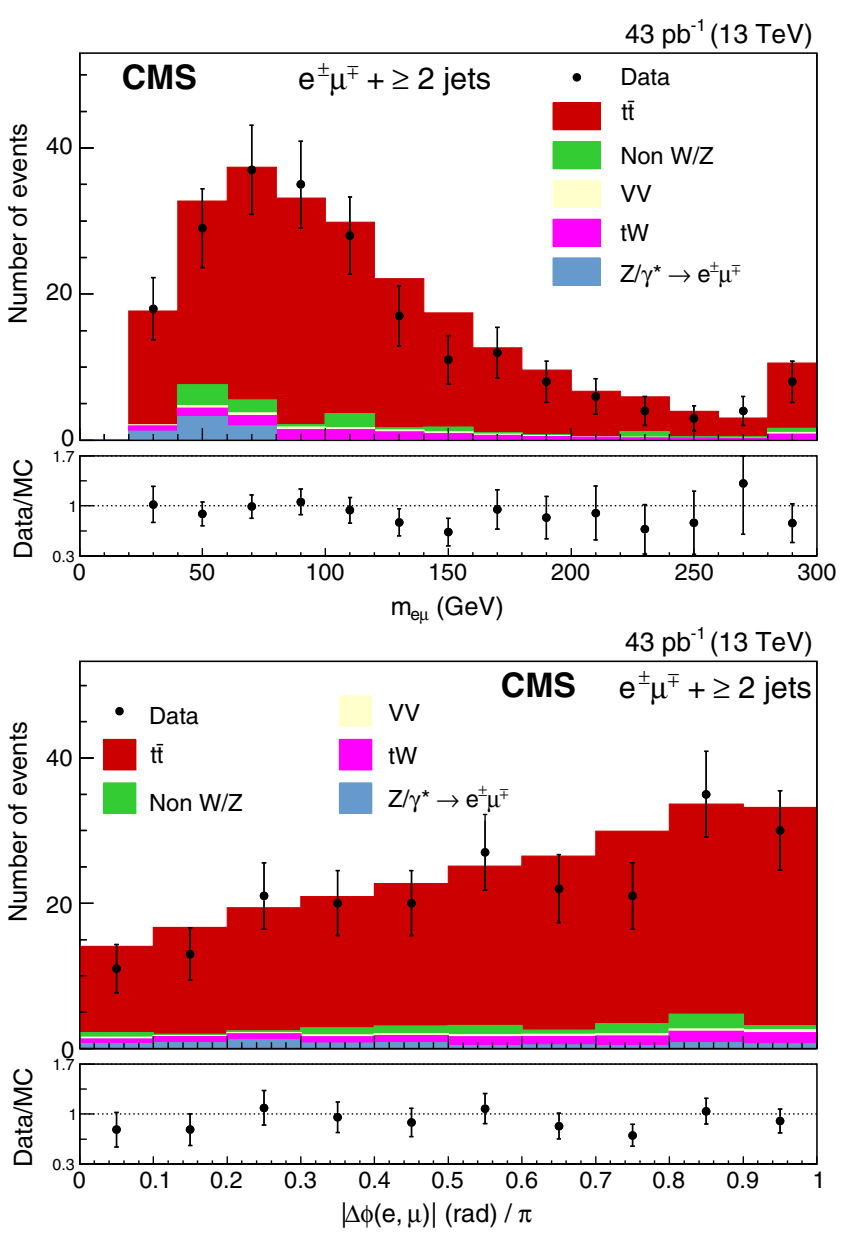

FIG. 2. The distributions in (top) the dilepton invariant mass, and (bottom) the difference in the azimuthal angle between the two leptons after all selections. The last bin in (top) contains the overflow events. The ratios of data to the sum of the expected yields are given at the bottom of each panel.

the invariant dilepton mass $m_{e \mu}$, which is sensitive to the existence of a new heavy object decaying into a $t \bar{t}$ pair. Figure 2 (bottom) shows the difference in azimuthal angle between the two leptons, $\Delta \phi(e, \mu)$, and explores the correlation between the $t$ and $\bar{t}$ spins [61-66]. For both distributions, data are in agreement with the SM expectations.

The dominant uncertainty is due to the preliminary integrated luminosity, which is estimated from $x-y$ beambeam scans performed in July 2015 utilizing the methods of Ref. [67]. The resulting uncertainty in the integrated luminosity is $4.8 \%$.

Smaller uncertainties arise from the measured trigger efficiency, and the lepton identification and isolation efficiencies. After the offline dilepton selection, the trigger efficiency is measured in data to be $(91 \pm 4) \%$ using triggers based on the $p_{\mathrm{T}}$ imbalance in the event. This efficiency is applied to the MC simulations and the uncertainty is taken as a global uncertainty. The uncertainties on the electron and muon identification and isolation 
efficiencies are estimated by changing the $p_{\mathrm{T}^{-}}$and $\eta^{-}$ dependent $\mathrm{SF}$ values by one standard deviation $( \pm 1 \sigma)$. The modeling of lepton energy scales is studied using $\mathrm{Z} \rightarrow e e$ and $\mu \mu$ events in data and in simulation, yielding an uncertainty in the electron energy scale of $1 \%$, and in the muon energy scale of $0.5 \%$. The impact of the uncertainty in the jet energy scale (JES) is estimated by changing the $p_{\mathrm{T}}$-and $\eta$-dependent JES SF by $\pm 1 \sigma$, and the uncertainty in jet energy resolution (JER) uncertainty is estimated through similar $\eta$-dependent $\pm 1 \sigma$ changes in the JER SF. The maximum of each of the deviations is taken as the uncertainty.

The distribution of the number of vertices per beam crossing is compared between data and simulation. The results indicate agreement of the total $p p$ inelastic cross section within $10 \%$. The result of varying this cross section by $\pm 10 \%$ for all MC samples is used to obtain the systematic uncertainty due to pileup.

Theory uncertainties on $t \bar{t}$ production involve the systematic bias related to the missing higher-order diagrams in POWHEG, and is estimated through studies of the signal acceptance by changing the renormalization and factorization scales in POWHEG simultaneously within the range $[\mu / 2,2 \mu]\left(\mu=\mu_{\mathrm{R}}=\mu_{\mathrm{F}}\right)$. In addition, the predictions of the NLO generators MG5_AMC@NLO (v5_2.2.2) and POWHEG are compared for $t \bar{t}$ production, where both use PYTHIA (v8.205) for hadronization, parton showering, and simulation of the underlying event. The uncertainty arising from the hadronization model mainly affects the JES and the fragmentation of jets. The uncertainty in the JES already contains a contribution from the uncertainty in the hadronization. The hadronization uncertainty is also determined by comparing samples of events generated with POWHEG, where the hadronization is either modeled with PYTHIA (v8.205) or HERWIG++ (v2.7.1). This also includes differences in parton showering, and the underlying event, and is called $t \bar{t}$ modeling uncertainty. All theory uncertainties on $t \bar{t}$ production are taken as the maximum difference found in the results. The uncertainty from the choice of PDF is determined by reweighting the sample of simulated $t \bar{t}$ events according to the 26 CT10 NLO [49,50] and the 100 NNPDF3.0 sets [25] of PDF uncertainties.

An uncertainty of 30\% in cross sections for $t W$ and $V V$ backgrounds are taken from measurements [68-76]. For DY production, a global cross section uncertainty of $15 \%$ is applied, which is derived from the variation of the SF for events passing the dilepton criteria and events passing all selection cuts. The systematic uncertainty in the estimated nonprompt lepton background is given mainly by the systematic uncertainty in the ratio of OS to SS events with misidentified leptons in the MC simulations. We checked how well the simulation models the production of misidentified leptons by examining additional control regions, with the observed discrepancy used to assign an uncertainty of $23 \%$ to the method.
TABLE I. Summary of individual contributions to the systematic uncertainty in the $\sigma_{t \bar{t}}$ measurement. The uncertainties are given in $\mathrm{pb}$, and as relative uncertainties. The separate total systematic uncertainty without integrated luminosity, the part attributed to the integrated luminosity, and the statistical contributions are added in quadrature to obtain the total uncertainty.

\begin{tabular}{lcc}
\hline \hline Source & $\Delta \sigma_{t \bar{t}}(\mathrm{pb})$ & $\Delta \sigma_{t \bar{t}} / \sigma_{t \bar{t}}(\%)$ \\
\hline Trigger efficiencies & 33 & 4.4 \\
Lepton efficiencies & 25 & 3.4 \\
Lepton energy scale & $<1$ & $\leq 0.1$ \\
Jet energy scale & 11 & 1.5 \\
Jet energy resolution & $<1$ & $\leq 0.1$ \\
Pileup & 5.2 & 0.7 \\
QCD scales & 1.4 & 0.2 \\
NLO generator of $t \bar{t}$ signal & 14 & 1.9 \\
Modeling of $t \bar{t}$ signal & 13 & 1.8 \\
PDF & 18 & 2.4 \\
Single top $t W$ background & 13 & 1.8 \\
$V V$ background & 3.5 & 0.5 \\
Drell-Yan background & 4.1 & 0.5 \\
Nonprompt leptons background & 7.6 & 1.0 \\
Total systematic (w/o luminosity) & 53 & 7.2 \\
Integrated luminosity & 36 & 4.8 \\
Statistical uncertainty & 58 & 7.8 \\
Total & 87 & 12 \\
\hline \hline
\end{tabular}

Table I summarizes the magnitude of the statistical and systematic uncertainties from different sources contributing to the $t \bar{t}$ production cross section. All sources of uncertainties are added in quadrature.

Table II shows the total number of events observed in data, together with the total number of background events expected from simulation or estimated from data. The mean acceptance multiplied by the selection efficiency and the branching fraction, as estimated from simulation at $m_{t}=172.5 \mathrm{GeV}$, is $\epsilon=(0.60 \pm 0.04) \%$, including statistical and systematic uncertainties. The measured fiducial cross section for $t \bar{t}$ production with two leptons (one electron and one muon) in the range $p_{\mathrm{T}}>20 \mathrm{GeV}$ and $|\eta|<2.4 \quad$ is $\quad \sigma_{t \bar{t}}^{\text {fid }}=12.4 \pm 1.0$ (stat) \pm 1.0 (syst) \pm 0.6 (lumi) pb. After applying all corrections, the inclusive

TABLE II. The number of $e \mu$ events after final event selection expected for background, and observed in data. The uncertainties represent the statistical and systematic components added in quadrature.

\begin{tabular}{lc}
\hline \hline Source & Number of events $e^{ \pm} \mu^{\mp}$ \\
\hline Drell-Yan & $6.9 \pm 1.2$ \\
Nonprompt leptons & $8.5 \pm 4.4$ \\
$t W$ & $10.9 \pm 3.4$ \\
$V V$ & $2.7 \pm 0.9$ \\
Total background & $29.1 \pm 5.7$ \\
Data & 220 \\
\hline \hline
\end{tabular}


cross section is measured to be $\sigma_{t \bar{t}}=746 \pm 58$ (stat) \pm 53 (syst) \pm 36 (lumi) pb.

A linear parametrization of the acceptance dependence on $m_{t}$ in the range $169.5-175.5 \mathrm{GeV}$ results in a cross section reduction of $\approx 0.7 \%$ at $m_{t}=173.34 \mathrm{GeV}$, the current world average of the top quark mass [24].

In an alternative analysis, the selected sample is split into events with $0,1,2$, and $>2 b$ quark jets, and $0,1,2$, and $>2$ additional light-flavor or gluon jets (i.e., not identified as b quark jets). Jets are identified as $b$ quark jets using the combined secondary vertex (CSV) algorithm [77]. A maximum likelihood fit of the yields in different input samples is performed to extract simultaneously $\sigma_{t \bar{t}}$ and the $b$ tagging efficiency. Systematic uncertainties are implemented through nuisance parameters [78]. This result is within $1 \%$ of the nominal analysis.

Figure 1 in the Supplemental Material [79] presents a summary of results for $\sigma_{t \bar{t}}$ from the combination of the Tevatron measurements at $1.96 \mathrm{TeV}$ [80], from CMS measurements at $\sqrt{s}=7$ and $8 \mathrm{TeV}[14,19]$, and from the measurement presented here at $\sqrt{s}=13 \mathrm{TeV}$, compared to the NNLO + NNLL predictions as a function of $\sqrt{s}$ for $p \bar{p}$ and $p p$ collisions [44].

In summary, the first measurement of the $t \bar{t}$ production cross section in proton-proton collisions at $\sqrt{s}=13 \mathrm{TeV}$ is presented for events containing an electron-muon pair and at least two jets. The measurement is obtained through an event-counting analysis based on a data sample corresponding to an integrated luminosity of $43 \mathrm{pb}^{-1}$. The result is $\sigma_{\bar{t} \bar{t}}=746 \pm 58$ (stat) \pm 53 (syst) \pm 36 (lumi) pb, with a total relative uncertainty of $12 \%$. This measurement is consistent with the SM prediction of $\sigma_{t \bar{t}}^{\mathrm{NNLO}+\mathrm{NNLL}}=$ $832_{-46}^{+40} \mathrm{pb}$ for a top quark mass of $172.5 \mathrm{GeV}$.

We congratulate our colleagues in the CERN accelerator departments for the excellent performance of the LHC and thank the technical and administrative staffs at CERN and at other CMS institutes for their contributions to the success of the CMS effort. In addition, we gratefully acknowledge the computing centers and personnel of the Worldwide LHC Computing Grid for delivering so effectively the computing infrastructure essential to our analyses. Finally, we acknowledge the enduring support for the construction and operation of the LHC and the CMS detector provided by the following funding agencies: BMWFW and FWF (Austria); FNRS and FWO (Belgium); CNPq, CAPES, FAPERJ, and FAPESP (Brazil); MES (Bulgaria); CERN; CAS, MoST, and NSFC (China); COLCIENCIAS (Colombia); MSES and CSF (Croatia); RPF (Cyprus); MoER, ERC IUT and ERDF (Estonia); Academy of Finland, MEC, and HIP (Finland); CEA and CNRS/IN2P3 (France); BMBF, DFG, and HGF (Germany); GSRT (Greece); OTKA and NIH (Hungary); DAE and DST (India); IPM (Iran); SFI (Ireland); INFN (Italy); MSIP and NRF (Republic of Korea); LAS (Lithuania); MOE and UM (Malaysia); CINVESTAV, CONACYT, SEP, and
UASLP-FAI (Mexico); MBIE (New Zealand); PAEC (Pakistan); MSHE and NSC (Poland); FCT (Portugal); JINR (Dubna); MON, RosAtom, RAS and RFBR (Russia); MESTD (Serbia); SEIDI and CPAN (Spain); Swiss Funding Agencies (Switzerland); MST (Taipei); ThEPCenter, IPST, STAR and NSTDA (Thailand); TUBITAK and TAEK (Turkey); NASU and SFFR (Ukraine); STFC (United Kingdom); DOE and NSF (USA).

[1] ATLAS Collaboration, Measurement of the top pair production cross section in $8 \mathrm{TeV}$ proton-proton collisions using kinematic information in the lepton + jets final state with ATLAS, Phys. Rev. D 91, 112013 (2015).

[2] ATLAS Collaboration, Differential top-antitop cross-section measurements as a function of observables constructed from final-state particles using pp collisions at $\sqrt{s}=$ $7 \mathrm{TeV}$ in the ATLAS detector, J. High Energy Phys. 06 (2015) 100.

[3] ATLAS Collaboration, Measurement of the $t \bar{t}$ production cross-section as a function of jet multiplicity and jet transverse momentum in $7 \mathrm{TeV}$ proton-proton collisions with the ATLAS detector, J. High Energy Phys. 01 (2015) 020.

[4] ATLAS Collaboration, Simultaneous measurements of the $t \bar{t}$, and $\mathrm{Z} / \gamma^{*} \rightarrow \tau \tau$ production cross sections in $p p$ collisions at $\sqrt{s}=7 \mathrm{TeV}$ with the ATLAS detector, Phys. Rev. D 91, 052005 (2015).

[5] ATLAS Collaboration, Measurements of normalized differential cross sections for $t \bar{t}$ production in $p p$ collisions at $\sqrt{s}=7 \mathrm{TeV}$ using the ATLAS detector, Phys. Rev. D 90, 072004 (2014).

[6] ATLAS Collaboration, Measurement of the $\bar{t} \bar{t}$ production cross-section using $e \mu$ events with $b$-tagged jets in $p p$ collisions at $\sqrt{s}=7$ and $8 \mathrm{TeV}$ with the ATLAS detector, Eur. Phys. J. C 74, 3109 (2014).

[7] ATLAS Collaboration, Measurement of the $t \bar{t}$ production cross section in the $\tau+$ jets channel using the ATLAS detector, Eur. Phys. J. C 73, 2328 (2013).

[8] ATLAS Collaboration, Measurement of the top quark pair cross section with ATLAS in $p p$ collisions at $\sqrt{s}=7 \mathrm{TeV}$ using final states with an electron or a muon and a hadronically decaying $\tau$ lepton, Phys. Lett. B 717, 89 (2012).

[9] ATLAS Collaboration, Measurement of $t \bar{t}$ production with a veto on additional central jet activity in $p p$ collisions at $\sqrt{s}=7 \mathrm{TeV}$ using the ATLAS detector, Eur. Phys. J. C 72, 2043 (2012).

[10] ATLAS Collaboration, Measurement of the cross section for top-quark pair production in $p p$ collisions at $\sqrt{s}=7 \mathrm{TeV}$ with the ATLAS detector using final states with two high- $p_{\mathrm{T}}$ leptons, J. High Energy Phys. 05 (2012) 059.

[11] ATLAS Collaboration, Measurement of the top quark pair production cross-section with ATLAS in the single lepton channel, Phys. Lett. B 711, 244 (2012).

[12] CMS Collaboration, Measurement of the differential cross section for top quark pair production in $p p$ collisions at $\sqrt{s}=8 \mathrm{TeV}$, Eur. Phys. J. C 75, 542 (2015). 
[13] CMS Collaboration, Measurement of the $t \bar{t}$ production cross section in $p p$ collisions at $\sqrt{s}=8 \mathrm{TeV}$ in dilepton final states containing one $\tau$ lepton, Phys. Lett. B 739, 23 (2014).

[14] CMS Collaboration, Measurement of the $t \bar{t}$ production cross section in the dilepton channel in $p p$ collisions at $\sqrt{s}=8 \mathrm{TeV}$, J. High Energy Phys. 02 (2014) 024

[15] CMS Collaboration, Measurement of the $t \bar{t}$ production cross section in the all-jet final state in $p p$ collisions at $\sqrt{s}=7 \mathrm{TeV}$, J. High Energy Phys. 05 (2013) 065.

[16] CMS Collaboration, Measurement of the $t \bar{t}$ production cross section in the $\tau+$ jets channel in $p p$ collisions at $\sqrt{s}=7 \mathrm{TeV}$, Eur. Phys. J. C 73, 2386 (2013).

[17] CMS Collaboration, Measurement of the $t \bar{t}$ production cross section in $p p$ collisions at $\sqrt{s}=7 \mathrm{TeV}$ with lepton + jets final states, Phys. Lett. B 720, 83 (2013).

[18] CMS Collaboration, Measurement of differential topquark-pair production cross sections in $p p$ colisions at $\sqrt{s}=7$ TeV, Eur. Phys. J. C 73, 2339 (2013).

[19] CMS Collaboration, Measurement of the $t \bar{t}$ production cross section in the dilepton channel in $p p$ collisions at $\sqrt{s}=7 \mathrm{TeV}$, J. High Energy Phys. 11 (2012) 067.

[20] CMS Collaboration, Measurement of the $t \bar{t}$ production cross section in $p p$ collisions at $\sqrt{s}=7 \mathrm{TeV}$ in dilepton final states containing a $\tau$, Phys. Rev. D 85, 112007 (2012).

[21] CMS Collaboration, The CMS experiment at the CERN LHC, J. Instrum. 3, S08004 (2008).

[22] S. Frixione, P. Nason, and C. Oleari, Matching NLO QCD computations with parton shower simulations: The POWHEG method, J. High Energy Phys. 11 (2007) 070.

[23] S. Alioli, P. Nason, C. Oleari, and E. Re, A general framework for implementing NLO calculations in shower Monte Carlo programs: The POWHEG BOX, J. High Energy Phys. 06 (2010) 043.

[24] ATLAS, CDF, CMS and D0 collaborations, First combination of Tevatron and LHC measurements of the top-quark mass, arXiv:1403.4427.

[25] F. Demartin, S. Forte, E. Mariani, J. Rojo, and A. Vicini, Impact of parton distribution function and $\alpha_{S}$ uncertainties on Higgs boson production in gluon fusion at hadron colliders, Phys. Rev. D 82, 014002 (2010).

[26] T. Sjöstrand, S. Mrenna, and P. Skands, PYTHIA 6.4 physics and manual, J. High Energy Phys. 05 (2006) 026.

[27] T. Sjöstrand, S. Ask, J. R. Christiansen, R. Corke, N. Desai, P. Ilten, S. Mrenna, S. Prestel, C. O. Rasmussen, and P. Skands, An introduction to PYTHIA 8.2, Comput. Phys. Commun. 191, 159 (2015).

[28] CMS Collaboration, Report No. CMS-PAS-GEN-14-001, 2014, https://cds.cern.ch/record/1697700.

[29] P. Skands, S. Carrazza, and J. Rojo, Tuning PYTHIA 8.1: The Monash 2013 tune, Eur. Phys. J. C 74, 3024 (2014).

[30] M. Bähr, S. Gieseke, M. A. Gigg, D. Grellscheid, K. Hamilton, O. Latunde-Dada, S. Plätzer, P. Richardson, M. H. Seymour, A. Sherstnev, and B. R. Webber, Herwig ++ physics and manual, Eur. Phys. J. C 58, 639 (2008).

[31] J. Alwall, R. Frederix, S. Frixione, V. Hirschi, F. Maltoni, O. Mattelaer, H. S. Shao, T. Stelzer, P. Torrielli, and M. Zaro, The automated computation of tree-level and next-to-leading order differential cross sections, and their matching to parton shower simulations, J. High Energy Phys. 07 (2014) 079.

[32] P. Artoisenet, R. Frederix, O. Mattelaer, and R. Rietkerk, Automatic spin-entangled decays of heavy resonances in Monte Carlo simulations, J. High Energy Phys. 03 (2013) 015.

[33] CMS Collaboration, Measurement of $t \bar{t}$ production with additional jet activity, including $b$ quark jets, in the dilepton channel using $p p$ collisions at $\sqrt{s}=8 \mathrm{TeV}$, arXiv: 1510.03072 .

[34] S. Alioli, P. Nason, C. Oleari, and E. Re, NLO single-top production matched with shower in POWHEG: $s$ - and t-channel contributions, J. High Energy Phys. 09 (2009) 111.

[35] E. Re, Single-top Wt-channel production matched with parton showers using the POWHEG method, Eur. Phys. J. C 71, 1547 (2011).

[36] N. Kidonakis, in Proceedings, Helmholtz International Summer School on Physics of Heavy Quarks and Hadrons (HQ 2013) (Verlag Deutsches Elektronen-Synchrotron, Hamburg, 2014), p. 139.

[37] J. M. Campbell and R. K. Ellis, MCFM for the Tevatron and the LHC, Nucl. Phys. B, Proc. Suppl. 205-206, 10 (2010).

[38] M. Czakon and A. Mitov, Top ++ : A program for the calculation of the top-pair cross-section at hadron colliders, Comput. Phys. Commun. 185, 2930 (2014).

[39] M. Beneke, P. Falgari, S. Klein, and C. Schwinn, Hadronic top-quark pair production with NNLL threshold resummation, Nucl. Phys. B855, 695 (2012).

[40] M. Cacciari, M. Czakon, M. Mangano, A. Mitov, and P. Nason, Top-pair production at hadron colliders with next-tonext-to-leading logarithmic soft-gluon resummation, Phys. Lett. B 710, 612 (2012).

[41] P. Baernreuther, Michal Czakon, and Alexander Mitov, Percent-Level-Precision Physics at the Tevatron: Next-toNext-to-Leading Order QCD Corrections to $q \bar{q} \rightarrow t \bar{t}+X$, Phys. Rev. Lett. 109, 132001 (2012).

[42] M. Czakon and A. Mitov, NNLO corrections to top-pair production at hadron colliders: The all-fermionic scattering channels, J. High Energy Phys. 12 (2012) 054.

[43] M. Czakon and A. Mitov, NNLO corrections to top pair production at hadron colliders: The quark-gluon reaction, J. High Energy Phys. 01 (2013) 080.

[44] M. Czakon, P. Fiedler, and A. Mitov, Total Top-Quark PairProduction Cross Section at Hadron Colliders Through $\mathrm{O}\left(\alpha_{S}^{4}\right)$, Phys. Rev. Lett. 110, 252004 (2013).

[45] M. Botje et al., The PDF4LHC Working Group Interim Recommendations, arXiv:1101.0538.

[46] S. Alekhin et al., The PDF4LHC Working Group Interim Report, arXiv:1101.0536.

[47] A. D. Martin, W. J. Stirling, R. S. Thorne, and G. Watt, Parton distributions for the LHC, Eur. Phys. J. C 63, 189 (2009).

[48] A. D. Martin, W. J. Stirling, R. S. Thorne, and G. Watt, Uncertainties on $\alpha_{s}$ in global PDF analyses and implications for predicted hadronic cross sections, Eur. Phys. J. C 64, 653 (2009).

[49] H.-L. Lai, M. Guzzi, J. Huston, Z. Li, P. M. Nadolsky, J. Pumplin, and C.P. Yuan, New parton distributions for collider physics, Phys. Rev. D 82, 074024 (2010). 
[50] J. Gao, M. Guzzi, J. Huston, H.-L. Lai, Z. Li, P. Nadolsky, J. Pumplin, D. Stump, and C.P. Yuan, CT10 next-to-next-to-leading order global analysis of QCD, Phys. Rev. D 89, 033009 (2014).

[51] R. D. Ball et al. (NNPDF), Parton distributions with LHC data, Nucl. Phys. B867, 244 (2013).

[52] CMS Collaboration, Report No. CMS-PAS-PFT-09-001, 2009, http://cdsweb.cern.ch/record/1194487.

[53] CMS Collaboration, Report No. CMS-PAS-PFT-10-001, 2010, http://cdsweb.cern.ch/record/1247373.

[54] CMS Collaboration, Performance of electron reconstruction and selection with the CMS detector in proton-proton collisions at $\sqrt{s}=8 \mathrm{TeV}$, J. Instrum. 10, P06005 (2015).

[55] CMS Collaboration, The performance of the CMS muon detector in proton-proton collisions at $\sqrt{s}=7 \mathrm{TeV}$ at the LHC, J. Instrum. 8, P11002 (2013).

[56] CMS Collaboration, Measurements of inclusive $W$ and $Z$ cross sections in $p p$ collisions at $\sqrt{s}=7 \mathrm{TeV}$, J. High Energy Phys. 01 (2011) 080.

[57] M. Cacciari, G. P. Salam, and G. Soyez, The anti- $k_{t}$ jet clustering algorithm, J. High Energy Phys. 04 (2008) 063.

[58] CMS Collaboration, Determination of jet energy calibration and transverse momentum resolution in CMS, J. Instrum. 6, P11002 (2011).

[59] CMS Collaboration, Measurement of the $t \bar{t}$ production cross section and the top quark mass in the dilepton channel in $p p$ collisions at $\sqrt{s}=7 \mathrm{TeV}$, J. High Energy Phys. 07 (2011) 049.

[60] CMS Collaboration, First measurement of the cross section for top-quark pair production in proton-proton collisions at $\sqrt{s}=7 \mathrm{TeV}$, Phys. Lett. B 695, 424 (2011).

[61] G. Mahlon and S. J. Parke, Spin correlation effects in top quark pair production at the LHC, Phys. Rev. D 81, 074024 (2010).

[62] W. Bernreuther and Z.-G. Si, Top quark spin correlations and polarization at the LHC: Standard model predictions and effects of anomalous top chromo moments, Phys. Lett. B 725, 115 (2013).

[63] ATLAS Collaboration, Measurement of Spin Correlation in Top-Antitop Quark Events and Search for Top Squark Pair Production in $p p$ Collisions at $\sqrt{s}=8 \mathrm{TeV}$ Using the ATLAS Detector, Phys. Rev. Lett. 114, 142001 (2015).

[64] ATLAS Collaboration, Measurements of spin correlation in top-antitop quark events from proton-proton collisions at $\sqrt{s}=7 \mathrm{TeV}$ using the ATLAS detector, Phys. Rev. D 90, 112016 (2014).

[65] ATLAS Collaboration, Observation of Spin Correlation in $t \bar{t}$ Events from $p p$ Collisions at $\sqrt{s}=7 \mathrm{TeV}$ using the ATLAS Detector, Phys. Rev. Lett. 108, 212001 (2012).

[66] CMS Collaboration, Measurements of $t \bar{t}$ Spin Correlations and Top-Quark Polarization Using Dilepton Final States in $p p$ Collisions at $\sqrt{s}=7 \mathrm{TeV}$, Phys. Rev. Lett. 112, 182001 (2014).

[67] CMS Collaboration, Report No. CMS-PAS-LUM-13-001, 2013, http://cdsweb.cern.ch/record/1643269.

[68] CMS Collaboration, Measurement of the $W^{+} W^{-}$and $Z Z$ production cross sections in $p p$ collisions at $\sqrt{s}=8 \mathrm{TeV}$, Phys. Lett. B 721, 190 (2013).

[69] CMS Collaboration, Measurement of the $W^{+} W^{-}$cross section in $p p$ collisions at $\sqrt{s}=7 \mathrm{TeV}$ and limits on anomalous $W W \gamma$ and $W W Z$ couplings, Eur. Phys. J. C 73, 2610 (2013).

[70] CMS Collaboration, Measurement of $W^{+} W^{-}$production and search for the Higgs boson in $p p$ collisions at $\sqrt{s}=7 \mathrm{TeV}$, Phys. Lett. B 699, 25 (2011).

[71] CMS Collaboration, Measurement of the sum of $W W$ and $W Z$ production with $W+$ dijet events in $p p$ collisions at $\sqrt{s}=7 \mathrm{TeV}$, Eur. Phys. J. C 73, 2283 (2013).

[72] CMS Collaboration, Measurement of the $Z Z$ production cross section and search for anomalous couplings in $2 \ell 2 \ell^{\prime}$ final states in $p p$ collisions at $\sqrt{s}=7 \mathrm{TeV}$, J. High Energy Phys. 01 (2013) 063.

[73] CMS Collaboration, Measurement of the single-top-quark $t$-channel cross section in $p p$ collisions at $\sqrt{s}=7 \mathrm{TeV}, \mathrm{J}$. High Energy Phys. 12 (2012) 035.

[74] ATLAS Collaboration, Measurement of the $W W$ cross section in $\sqrt{s}=7 \mathrm{TeV} p p$ collisions with the ATLAS detector and limits on anomalous gauge couplings, Phys. Lett. B 712, 289 (2012).

[75] ATLAS Collaboration, Measurement of the $W^{ \pm} Z$ production cross section and limits on anomalous triple gauge couplings in proton-proton collisions at $\sqrt{\mathrm{s}}=$ $7 \mathrm{TeV}$ with the ATLAS detector, Phys. Lett. B 709, 341 (2012).

[76] ATLAS Collaboration, Measurement of the $Z Z$ Production Cross Section and Limits on Anomalous Neutral Triple Gauge Couplings in Proton-Proton Collisions at $\sqrt{s}=$ $7 \mathrm{TeV}$ with the ATLAS Detector, Phys. Rev. Lett. 108, 041804 (2012).

[77] CMS Collaboration, Identification of $b$-quark jets with the CMS experiment, J. Instrum. 8, P04013 (2013).

[78] D. A. S. Fraser, N. Reid, and A. C. M. Wong, Inference for bounded parameters, Phys. Rev. D 69, 033002 (2004).

[79] See Supplemental Material at http://link.aps.org/ supplemental/10.1103/PhysRevLett.116.052002 for a figure of top-quark pair production cross section measurements as a function of the center-of-mass energy.

[80] CDF and D0 Collaborations, Combination of measurements of the top-quark pair production cross section from the Tevatron Collider, Phys. Rev. D 89, 072001 (2014).

V. Khachatryan, ${ }^{1}$ A. M. Sirunyan, ${ }^{1}$ A. Tumasyan, ${ }^{1}$ W. Adam, ${ }^{2}$ E. Asilar, ${ }^{2}$ T. Bergauer, ${ }^{2}$ J. Brandstetter, ${ }^{2}$ E. Brondolin, ${ }^{2}$ M. Dragicevic, ${ }^{2}$ J. Erö, ${ }^{2}$ M. Flechl, ${ }^{2}$ M. Friedl, ${ }^{2}$ R. Frühwirth, ${ }^{2, b}$ V. M. Ghete, ${ }^{2}$ C. Hartl, ${ }^{2}$ N. Hörmann, ${ }^{2}$ J. Hrubec, ${ }^{2}$ M. Jeitler, ${ }^{2, b}$ V. Knünz, ${ }^{2}$ A. König, ${ }^{2}$ M. Krammer, ${ }^{2, b}$ I. Krätschmer, ${ }^{2}$ D. Liko, ${ }^{2}$ T. Matsushita, ${ }^{2}$ I. Mikulec, ${ }^{2}$ D. Rabady, ${ }^{2, c}$ B. Rahbaran, ${ }^{2}$ H. Rohringer, ${ }^{2}$ J. Schieck, ${ }^{2, b}$ R. Schöfbeck, ${ }^{2}$ J. Strauss, ${ }^{2}$ W. Treberer-Treberspurg, ${ }^{2}$ W. Waltenberger, ${ }^{2}$ 
C.-E. Wulz, ${ }^{2, b}$ V. Mossolov, ${ }^{3}$ N. Shumeiko, ${ }^{3}$ J. Suarez Gonzalez, ${ }^{3}$ S. Alderweireldt, ${ }^{4}$ T. Cornelis, ${ }^{4}$ E. A. De Wolf, ${ }^{4}$ X. Janssen, ${ }^{4}$ A. Knutsson, ${ }^{4}$ J. Lauwers, ${ }^{4}$ S. Luyckx,${ }^{4}$ M. Van De Klundert, ${ }^{4}$ H. Van Haevermaet, ${ }^{4}$ P. Van Mechelen, ${ }^{4}$ N. Van Remortel, ${ }^{4}$ A. Van Spilbeeck, ${ }^{4}$ S. Abu Zeid, ${ }^{5}$ F. Blekman, ${ }^{5}$ J. D’Hondt, ${ }^{5}$ N. Daci, ${ }^{5}$ I. De Bruyn, ${ }^{5}$ K. Deroover, ${ }^{5}$

N. Heracleous, ${ }^{5}$ J. Keaveney, ${ }^{5}$ S. Lowette, ${ }^{5}$ L. Moreels, ${ }^{5}$ A. Olbrechts, ${ }^{5}$ Q. Python, ${ }^{5}$ D. Strom, ${ }^{5}$ S. Tavernier, ${ }^{5}$

W. Van Doninck, ${ }^{5}$ P. Van Mulders, ${ }^{5}$ G. P. Van Onsem, ${ }^{5}$ I. Van Parijs, ${ }^{5}$ P. Barria, ${ }^{6}$ H. Brun, ${ }^{6}$ C. Caillol, ${ }^{6}$ B. Clerbaux, ${ }^{6}$ G. De Lentdecker, ${ }^{6}$ G. Fasanella, ${ }^{6}$ L. Favart, ${ }^{6}$ A. Grebenyuk, ${ }^{6}$ G. Karapostoli, ${ }^{6}$ T. Lenzi, ${ }^{6}$ A. Léonard, ${ }^{6}$ T. Maerschalk, ${ }^{6}$ A. Marinov, ${ }^{6}$ L. Perniè, ${ }^{6}$ A. Randle-conde, ${ }^{6}$ T. Seva, ${ }^{6}$ C. Vander Velde, ${ }^{6}$ P. Vanlaer, ${ }^{6}$ R. Yonamine, ${ }^{6}$ F. Zenoni, ${ }^{6}$ F. Zhang, ${ }^{6, d}$

K. Beernaert, ${ }^{7}$ L. Benucci, ${ }^{7}$ A. Cimmino, ${ }^{7}$ S. Crucy, ${ }^{7}$ D. Dobur, ${ }^{7}$ A. Fagot, ${ }^{7}$ G. Garcia, ${ }^{7}$ M. Gul, ${ }^{7}$ J. Mccartin, ${ }^{7}$ A. A. Ocampo Rios, ${ }^{7}$ D. Poyraz, ${ }^{7}$ D. Ryckbosch, ${ }^{7}$ S. Salva, ${ }^{7}$ M. Sigamani, ${ }^{7}$ M. Tytgat, ${ }^{7}$ W. Van Driessche, ${ }^{7}$ E. Yazgan, ${ }^{7}$ N. Zaganidis, ${ }^{7}$ S. Basegmez, ${ }^{8}$ C. Beluffi,,${ }^{8, e}$ O. Bondu, ${ }^{8}$ S. Brochet, ${ }^{8}$ G. Bruno, ${ }^{8}$ A. Caudron, ${ }^{8}$ L. Ceard,${ }^{8}$ G. G. Da Silveira, ${ }^{8}$

C. Delaere, ${ }^{8}$ D. Favart, ${ }^{8}$ L. Forthomme, ${ }^{8}$ A. Giammanco, ${ }^{8, \mathrm{f}}$ J. Hollar, ${ }^{8}$ A. Jafari, ${ }^{8}$ P. Jez, ${ }^{8}$ M. Komm, ${ }^{8}$ V. Lemaitre, ${ }^{8}$ A. Mertens ${ }^{8}$ M. Musich,${ }^{8}$ C. Nuttens, ${ }^{8}$ L. Perrini, ${ }^{8}$ A. Pin, ${ }^{8}$ K. Piotrzkowski, ${ }^{8}$ A. Popov, ${ }^{8, g}$ L. Quertenmont, ${ }^{8}$ M. Selvaggi, ${ }^{8}$ M. Vidal Marono, ${ }^{8}$ N. Beliy, ${ }^{9}$ G. H. Hammad, ${ }^{9}$ W. L. Aldá Júnior, ${ }^{10}$ F. L. Alves, ${ }^{10}$ G. A. Alves, ${ }^{10}$ L. Brito, ${ }^{10}$ M. Correa Martins Junior, ${ }^{10}$ M. Hamer, ${ }^{10}$ C. Hensel, ${ }^{10}$ A. Moraes, ${ }^{10}$ M. E. Pol, ${ }^{10}$ P. Rebello Teles, ${ }^{10}$

E. Belchior Batista Das Chagas, ${ }^{11}$ W. Carvalho, ${ }^{11}$ J. Chinellato, ${ }^{11, h}$ A. Custódio, ${ }^{11}$ E. M. Da Costa, ${ }^{11}$ D. De Jesus Damiao, ${ }^{11}$ C. De Oliveira Martins, ${ }^{11}$ S. Fonseca De Souza, ${ }^{11}$ L. M. Huertas Guativa, ${ }^{11}$ H. Malbouisson, ${ }^{11}$ D. Matos Figueiredo, ${ }^{11}$ C. Mora Herrera ${ }^{11}$ L. Mundim, ${ }^{11}$ H. Nogima, ${ }^{11}$ W. L. Prado Da Silva, ${ }^{11}$ A. Santoro, ${ }^{11}$ A. Sznajder, ${ }^{11}$

E. J. Tonelli Manganote, ${ }^{11, h}$ A. Vilela Pereira, ${ }^{11}$ S. Ahuja, ${ }^{12 a}$ C. A. Bernardes, ${ }^{12 b}$ A. De Souza Santos, ${ }^{12 b}$ S. Dogra, ${ }^{12 a}$ T. R. Fernandez Perez Tomei, ${ }^{12 a}$ E. M. Gregores, ${ }^{12 b}$ P. G. Mercadante, ${ }^{12 b}$ C. S. Moon, ${ }^{12 a, i}$ S. F. Novaes, ${ }^{12 a}$ Sandra S. Padula, ${ }^{12 a}$ D. Romero Abad, ${ }^{12 a}$ J. C. Ruiz Vargas, ${ }^{12 a}$ A. Aleksandrov,${ }^{13}$ R. Hadjiiska, ${ }^{13}$ P. Iaydjiev, ${ }^{13}$ M. Rodozov, ${ }^{13}$ S. Stoykova, ${ }^{13}$ G. Sultanov,${ }^{13}$ M. Vutova, ${ }^{13}$ A. Dimitrov, ${ }^{14}$ I. Glushkov, ${ }^{14}$ L. Litov, ${ }^{14}$ B. Pavlov, ${ }^{14}$ P. Petkov, ${ }^{14}$ M. Ahmad, ${ }^{15}$ J. G. Bian, ${ }^{15}$ G. M. Chen,${ }^{15}$ H. S. Chen, ${ }^{15}$ M. Chen, ${ }^{15}$ T. Cheng, ${ }^{15}$ R. Du, ${ }^{15}$ C. H. Jiang, ${ }^{15}$ R. Plestina,${ }^{15, j}$ F. Romeo, ${ }^{15}$ S. M. Shaheen, ${ }^{15}$ A. Spiezia,${ }^{15}$ J. Tao, ${ }^{15}$ C. Wang, ${ }^{15}$ Z. Wang,${ }^{15}$ H. Zhang, ${ }^{15}$ C. Asawatangtrakuldee,${ }^{16}$ Y. Ban, ${ }^{16}$ Q. Li ${ }^{16}$ S. Liu ${ }^{16}$ Y. Mao, ${ }^{16}$ S. J. Qian, ${ }^{16}$ D. Wang,,${ }^{16}$ Z. Xu, ${ }^{16}$ C. Avila,${ }^{17}$ A. Cabrera, ${ }^{17}$ L. F. Chaparro Sierra, ${ }^{17}$ C. Florez, ${ }^{17}$ J. P. Gomez,${ }^{17}$ B. Gomez Moreno, ${ }^{17}$ J. C. Sanabria,${ }^{17}$ N. Godinovic, ${ }^{18}$ D. Lelas, ${ }^{18}$ I. Puljak, ${ }^{18}$ P. M. Ribeiro Cipriano, ${ }^{18}$ Z. Antunovic, ${ }^{19}$ M. Kovac, ${ }^{19}$ V. Brigljevic,${ }^{20}$ K. Kadija ${ }^{20}$ J. Luetic, ${ }^{20}$ S. Micanovic, ${ }^{20}$ L. Sudic, ${ }^{20}$ A. Attikis, ${ }^{21}$ G. Mavromanolakis, ${ }^{21}$ J. Mousa,${ }^{21}$ C. Nicolaou, ${ }^{21}$ F. Ptochos,${ }^{21}$ P. A. Razis ${ }^{21}$ H. Rykaczewski, ${ }^{21}$ M. Bodlak,${ }^{22}$ M. Finger, ${ }^{22, k}$ M. Finger Jr., ${ }^{22, \mathrm{k}}$ E. El-khateeb, ${ }^{23,1,1}$ T. Elkafrawy, ${ }^{23,1}$ A. Mohamed ${ }^{23, \mathrm{~m}}$ Y. Mohammed ${ }^{23, \mathrm{n}}$ E. Salama, ${ }^{23,0,1}$ B. Calpas, ${ }^{24}$ M. Kadastik, ${ }^{24}$ M. Murumaa, ${ }^{24}$ M. Raidal,${ }^{24}$ A. Tiko, ${ }^{24}$ C. Veelken, ${ }^{24}$ P. Eerola, ${ }^{25}$ J. Pekkanen, ${ }^{25}$ M. Voutilainen, ${ }^{25}$ J. Härkönen, ${ }^{26}$ V. Karimäki, ${ }^{26}$ R. Kinnunen, ${ }^{26}$ T. Lampén, ${ }^{26}$ K. Lassila-Perini, ${ }^{26}$ S. Lehti, ${ }^{26}$ T. Lindén, ${ }^{26}$ P. Luukka, ${ }^{26}$ T. Mäenpää, ${ }^{26}$ T. Peltola, ${ }^{26}$ E. Tuominen, ${ }^{26}$ J. Tuominiemi, ${ }^{26}$ E. Tuovinen, ${ }^{26}$ L. Wendland, ${ }^{26}$ J. Talvitie,${ }^{27}$ T. Tuuva, ${ }^{27}$ M. Besancon, ${ }^{28}$ F. Couderc, ${ }^{28}$ M. Dejardin, ${ }^{28}$ D. Denegri, ${ }^{28}$ B. Fabbro, ${ }^{28}$ J. L. Faure, ${ }^{28}$ C. Favaro, ${ }^{28}$ F. Ferri, ${ }^{28}$ S. Ganjour,${ }^{28}$ A. Givernaud, ${ }^{28}$ P. Gras, ${ }^{28}$ G. Hamel de Monchenault, ${ }^{28}$ P. Jarry, ${ }^{28}$ E. Locci, ${ }^{28}$ M. Machet, ${ }^{28}$ J. Malcles, ${ }^{28}$ J. Rander, ${ }^{28}$ A. Rosowsky, ${ }^{28}$ M. Titov, ${ }^{28}$ A. Zghiche, ${ }^{28}$ I. Antropov, ${ }^{29}$ S. Baffioni, ${ }^{29}$ F. Beaudette, ${ }^{29}$ P. Busson, ${ }^{29}$ L. Cadamuro, ${ }^{29}$ E. Chapon, ${ }^{29}$ C. Charlot ${ }^{29}$ T. Dahms,${ }^{29}$ O. Davignon, ${ }^{29}$ N. Filipovic, ${ }^{29}$ R. Granier de Cassagnac, ${ }^{29}$ M. Jo, ${ }^{29}$ S. Lisniak, ${ }^{29}$ L. Mastrolorenzo, ${ }^{29}$ P. Miné, ${ }^{29}$ I. N. Naranjo, ${ }^{29}$ M. Nguyen, ${ }^{29}$ C. Ochando, ${ }^{29}$ G. Ortona,${ }^{29}$ P. Paganini, ${ }^{29}$ P. Pigard, ${ }^{29}$ S. Regnard ${ }^{29}$ R. Salerno, ${ }^{29}$ J. B. Sauvan, ${ }^{29}$ Y. Sirois, ${ }^{29}$ T. Strebler, ${ }^{29}$ Y. Yilmaz,${ }^{29}$ A. Zabi, ${ }^{29}$ J.-L. Agram, ${ }^{30, p}$ J. Andrea, ${ }^{30}$ A. Aubin, ${ }^{30}$ D. Bloch ${ }^{30}$ J.-M. Brom, ${ }^{30}$ M. Buttignol, ${ }^{30}$ E. C. Chabert, ${ }^{30}$ N. Chanon, ${ }^{30}$ C. Collard,${ }^{30}$ E. Conte,${ }^{30, p}$ X. Coubez,${ }^{30}$ J.-C. Fontaine, ${ }^{30, p}$ D. Gelé, ${ }^{30}$ U. Goerlach ${ }^{30}$ C. Goetzmann, ${ }^{30}$ A.-C. Le Bihan, ${ }^{30}$ J. A. Merlin, ${ }^{30, c}$ K. Skovpen, ${ }^{30}$ P. Van Hove, ${ }^{30}$ S. Gadrat,${ }^{31}$ S. Beauceron, ${ }^{32}$ C. Bernet, ${ }^{32}$ G. Boudoul, ${ }^{32}$ E. Bouvier, ${ }^{32}$ C. A. Carrillo Montoya, ${ }^{32}$ R. Chierici, ${ }^{32}$ D. Contardo, ${ }^{32}$ B. Courbon, ${ }^{32}$ P. Depasse, ${ }^{32}$ H. El Mamouni, ${ }^{32}$ J. Fan, ${ }^{32}$ J. Fay, ${ }^{32}$ S. Gascon, ${ }^{32}$ M. Gouzevitch, ${ }^{32}$ B. Ille, ${ }^{32}$ F. Lagarde, ${ }^{32}$ I. B. Laktineh, ${ }^{32}$ M. Lethuillier, ${ }^{32}$ L. Mirabito, ${ }^{32}$ A. L. Pequegnot, ${ }^{32}$ S. Perries, ${ }^{32}$ J. D. Ruiz Alvarez,${ }^{32}$ D. Sabes, ${ }^{32}$ L. Sgandurra, ${ }^{32}$ V. Sordini, ${ }^{32}$ M. Vander Donckt, ${ }^{32}$ P. Verdier, ${ }^{32}$ S. Viret,${ }^{32}$ T. Toriashvili, ${ }^{33, q}$ I. Bagaturia, ${ }^{34, r}$ C. Autermann, ${ }^{35}$ S. Beranek, ${ }^{35}$ L. Feld, ${ }^{35}$ A. Heister, ${ }^{35}$ M. K. Kiesel ${ }^{35}$ K. Klein, ${ }^{35}$ M. Lipinski, ${ }^{35}$ A. Ostapchuk, ${ }^{35}$ M. Preuten, ${ }^{35}$ F. Raupach, ${ }^{35}$ S. Schael,${ }^{35}$ J. F. Schulte, ${ }^{35}$ T. Verlage, ${ }^{35}$ H. Weber, ${ }^{35}$ B. Wittmer, ${ }^{35}$ V. Zhukov, ${ }^{35,9}$ M. Ata, ${ }^{36}$ M. Brodski, ${ }^{36}$ E. Dietz-Laursonn, ${ }^{36}$ D. Duchardt, ${ }^{36}$ M. Endres,${ }^{36}$ M. Erdmann, ${ }^{36}$ S. Erdweg, ${ }^{36}$ T. Esch ${ }^{36}$ R. Fischer,${ }^{36}$ A. Güth, ${ }^{36}$ T. Hebbeker,${ }^{36}$ C. Heidemann, ${ }^{36}$ K. Hoepfner, ${ }^{36}$ S. Knutzen, ${ }^{36}$ P. Kreuzer,${ }^{36}$ M. Merschmeyer, ${ }^{36}$ A. Meyer, ${ }^{36}$ P. Millet, ${ }^{36}$ M. Olschewski, ${ }^{36}$ K. Padeken, ${ }^{36}$ P. Papacz,${ }^{36}$ T. Pook, ${ }^{36}$ M. Radziej, ${ }^{36}$ H. Reithler, ${ }^{36}$ M. Rieger, ${ }^{36}$ F. Scheuch, ${ }^{36}$ L. Sonnenschein, ${ }^{36}$ D. Teyssier, ${ }^{36}$ S. Thüer, ${ }^{36}$ V. Cherepanov, ${ }^{37}$ Y. Erdogan, ${ }^{37}$ 
G. Flügge,${ }^{37}$ H. Geenen, ${ }^{37}$ M. Geisler,${ }^{37}$ F. Hoehle, ${ }^{37}$ B. Kargoll, ${ }^{37}$ T. Kress,${ }^{37}$ Y. Kuessel, ${ }^{37}$ A. Künsken,${ }^{37}$ J. Lingemann, ${ }^{37}$ A. Nehrkorn, ${ }^{37}$ A. Nowack,${ }^{37}$ I. M. Nugent,${ }^{37}$ C. Pistone,${ }^{37}$ O. Pooth,${ }^{37}$ A. Stahl,${ }^{37}$ M. Aldaya Martin, ${ }^{38}$ T. Arndt,${ }^{38}$ I. Asin,${ }^{38}$ N. Bartosik, ${ }^{38}$ O. Behnke, ${ }^{38}$ U. Behrens, ${ }^{38}$ A. J. Bell, ${ }^{38}$ K. Borras, ${ }^{38, s}$ A. Burgmeier, ${ }^{38}$ A. Campbell, ${ }^{38}$ S. Choudhury,${ }^{38, t}$ F. Costanza, ${ }^{38}$ C. Diez Pardos, ${ }^{38}$ G. Dolinska, ${ }^{38}$ S. Dooling, ${ }^{38}$ T. Dorland, ${ }^{38}$ G. Eckerlin, ${ }^{38}$ D. Eckstein,${ }^{38}$ T. Eichhorn, ${ }^{38}$ G. Flucke, ${ }^{38}$ E. Gallo, ${ }^{38, u}$ J. Garay Garcia, ${ }^{38}$ A. Geiser,${ }^{38}$ A. Gizhko, ${ }^{38}$ A. Grohsjean, ${ }^{38}$ P. Gunnellini, ${ }^{38}$ A. Harb,${ }^{38}$ J. Hauk, ${ }^{38}$ M. Hempel, ${ }^{38, v}$ H. Jung, ${ }^{38}$ A. Kalogeropoulos ${ }^{38}$ O. Karacheban, ${ }^{38, v}$ M. Kasemann, ${ }^{38}$ P. Katsas, ${ }^{38}$ J. Kieseler, ${ }^{38}$ C. Kleinwort, ${ }^{38}$ I. Korol, ${ }^{38}$ W. Lange, ${ }^{38}$ J. Leonard, ${ }^{38}$ K. Lipka, ${ }^{38}$ A. Lobanov, ${ }^{38}$ W. Lohmann, ${ }^{38, v}$ R. Mankel, ${ }^{38}$ I. Marfin,,${ }^{38, v}$ I.-A. Melzer-Pellmann, ${ }^{38}$ A. B. Meyer,${ }^{38}$ G. Mittag, ${ }^{38}$ J. Mnich, ${ }^{38}$ A. Mussgiller, ${ }^{38}$ S. Naumann-Emme, ${ }^{38}$ A. Nayak ${ }^{38}$ E. Ntomari, ${ }^{38}$ H. Perrey, ${ }^{38}$ D. Pitzl, ${ }^{38}$ R. Placakyte, ${ }^{38}$ A. Raspereza, ${ }^{38}$ B. Roland ${ }^{38}$ M. Ö. Sahin,${ }^{38}$ M. Savitskyi, ${ }^{38}$ P. Saxena, ${ }^{38}$ T. Schoerner-Sadenius, ${ }^{38}$ M. Schröder, ${ }^{38}$ C. Schwanenberger, ${ }^{38}$ C. Seitz, ${ }^{38}$ S. Spannagel, ${ }^{38}$ K. D. Trippkewitz, ${ }^{38}$

R. Walsh, ${ }^{38}$ C. Wissing, ${ }^{38}$ V. Blobel,${ }^{39}$ M. Centis Vignali, ${ }^{39}$ A. R. Draeger, ${ }^{39}$ J. Erfle, ${ }^{39}$ E. Garutti, ${ }^{39}$ K. Goebel, ${ }^{39}$

D. Gonzalez, ${ }^{39}$ M. Görner, ${ }^{39}$ J. Haller, ${ }^{39}$ M. Hoffmann, ${ }^{39}$ R. S. Höing, ${ }^{39}$ A. Junkes, ${ }^{39}$ R. Klanner, ${ }^{39}$ R. Kogler, ${ }^{39}$ N. Kovalchuk, ${ }^{39}$ T. Lapsien, ${ }^{39}$ T. Lenz, ${ }^{39}$ I. Marchesini, ${ }^{39}$ D. Marconi, ${ }^{39}$ M. Meyer, ${ }^{39}$ D. Nowatschin, ${ }^{39}$ J. Ott, ${ }^{39}$ F. Pantaleo, ${ }^{39, c}$ T. Peiffer, ${ }^{39}$ A. Perieanu, ${ }^{39}$ N. Pietsch,,${ }^{39}$ J. Poehlsen, ${ }^{39}$ D. Rathjens, ${ }^{39}$ C. Sander, ${ }^{39}$ C. Scharf, ${ }^{39}$ H. Schettler, ${ }^{39}$ P. Schleper, ${ }^{39}$ E. Schlieckau, ${ }^{39}$ A. Schmidt, ${ }^{39}$ J. Schwandt, ${ }^{39}$ V. Sola, ${ }^{39}$ H. Stadie, ${ }^{39}$ G. Steinbrück,,${ }^{39}$ H. Tholen, ${ }^{39}$ D. Troendle, ${ }^{39}$ E. Usai, ${ }^{39}$ L. Vanelderen,${ }^{39}$ A. Vanhoefer ${ }^{39}$ B. Vormwald, ${ }^{39}$ C. Barth, ${ }^{40}$ C. Baus,${ }^{40}$ J. Berger, ${ }^{40}$ C. Böser, ${ }^{40}$ E. Butz ${ }^{40}$ T. Chwalek,${ }^{40}$ F. Colombo,${ }^{40}$ W. De Boer, ${ }^{40}$ A. Descroix,${ }^{40}$ A. Dierlamm, ${ }^{40}$ S. Fink, ${ }^{40}$ F. Frensch,${ }^{40}$ R. Friese,${ }^{40}$ M. Giffels, ${ }^{40}$ A. Gilbert, ${ }^{40}$ D. Haitz, ${ }^{40}$ F. Hartmann, ${ }^{40, c}$ S. M. Heindl, ${ }^{40}$ U. Husemann, ${ }^{40}$ I. Katkov, ${ }^{40, g}$ A. Kornmayer, ${ }^{40, c}$ P. Lobelle Pardo ${ }^{40}$ B. Maier, ${ }^{40}$ H. Mildner, ${ }^{40}$ M. U. Mozer, ${ }^{40}$ T. Müller, ${ }^{40}$ Th. Müller, ${ }^{40}$ M. Plagge,${ }^{40}$ G. Quast, ${ }^{40}$ K. Rabbertz, ${ }^{40}$ S. Röcker, ${ }^{40}$ F. Roscher, ${ }^{40}$ G. Sieber, ${ }^{40}$ H. J. Simonis,${ }^{40}$ F. M. Stober, ${ }^{40}$ R. Ulrich, ${ }^{40}$ J. Wagner-Kuhr, ${ }^{40}$ S. Wayand, ${ }^{40} \mathrm{M}$. Weber, ${ }^{40}$ T. Weiler, ${ }^{40} \mathrm{~S}$. Williamson, ${ }^{40} \mathrm{C}$. Wöhrmann, ${ }^{40}$ R. Wolf, ${ }^{40}$ G. Anagnostou, ${ }^{41}$ G. Daskalakis,${ }^{41}$ T. Geralis, ${ }^{41}$ V. A. Giakoumopoulou, ${ }^{41}$ A. Kyriakis, ${ }^{41}$ D. Loukas, ${ }^{41}$ A. Psallidas, ${ }^{41}$ I. Topsis-Giotis, ${ }^{41}$ A. Agapitos, ${ }^{42}$ S. Kesisoglou, ${ }^{42}$ A. Panagiotou, ${ }^{42}$ N. Saoulidou, ${ }^{42}$ E. Tziaferi, ${ }^{42}$ I. Evangelou, ${ }^{43}$ G. Flouris, ${ }^{43}$ C. Foudas, ${ }^{43}$ P. Kokkas, ${ }^{43}$ N. Loukas, ${ }^{43}$ N. Manthos ${ }^{43}$ I. Papadopoulos, ${ }^{43}$ E. Paradas, ${ }^{43}$ J. Strologas, ${ }^{43}$ G. Bencze, ${ }^{44}$ C. Hajdu, ${ }^{44}$ A. Hazi, ${ }^{44}$ P. Hidas, ${ }^{44}$ D. Horvath ${ }^{44, w}$ F. Sikler, ${ }^{44}$ V. Veszpremi, ${ }^{44}$ G. Vesztergombi, ${ }^{44, \mathrm{x}}$ A. J. Zsigmond ${ }^{44}$ N. Beni,${ }^{45}$ S. Czellar, ${ }^{45}$ J. Karancsi, ${ }^{45, y}$ J. Molnar, ${ }^{45}$ Z. Szillasi, ${ }^{45, c}$ M. Bartók, ${ }^{46, z}$ A. Makovec, ${ }^{46}$ P. Raics, ${ }^{46}$ Z. L. Trocsanyi, ${ }^{46}$ B. Ujvari, ${ }^{46}$ P. Mal, ${ }^{47}$ K. Mandal, ${ }^{47}$

D. K. Sahoo, ${ }^{47}$ N. Sahoo, ${ }^{47}$ S. K. Swain, ${ }^{47}$ S. Bansal,${ }^{48}$ S. B. Beri, ${ }^{48}$ V. Bhatnagar, ${ }^{48}$ R. Chawla, ${ }^{48}$ R. Gupta, ${ }^{48}$ U. Bhawandeep, ${ }^{48}$ A. K. Kalsi, ${ }^{48}$ A. Kaur, ${ }^{48}$ M. Kaur ${ }^{48}$ R. Kumar, ${ }^{48}$ A. Mehta, ${ }^{48}$ M. Mittal, ${ }^{48}$ J. B. Singh, ${ }^{48}$ G. Walia, ${ }^{48}$ Ashok Kumar, ${ }^{49}$ A. Bhardwaj, ${ }^{49}$ B. C. Choudhary, ${ }^{49}$ R. B. Garg, ${ }^{49}$ A. Kumar, ${ }^{49}$ S. Malhotra, ${ }^{49}$ M. Naimuddin, ${ }^{49}$ N. Nishu ${ }^{49}$ K. Ranjan, ${ }^{49}$ R. Sharma, ${ }^{49}$ V. Sharma, ${ }^{49}$ S. Bhattacharya,${ }^{50}$ K. Chatterjee, ${ }^{50}$ S. Dey, ${ }^{50}$ S. Dutta,${ }^{50}$ Sa. Jain,${ }^{50}$ N. Majumdar,${ }^{50}$ A. Modak, ${ }^{50}$ K. Mondal, ${ }^{50}$ S. Mukherjee, ${ }^{50}$ S. Mukhopadhyay, ${ }^{50}$ A. Roy, ${ }^{50}$ D. Roy, ${ }^{50}$ S. Roy Chowdhury, ${ }^{50}$ S. Sarkar, ${ }^{50}$ M. Sharan, ${ }^{50}$ A. Abdulsalam, ${ }^{51}$ R. Chudasama, ${ }^{51}$ D. Dutta, ${ }^{51}$ V. Jha, ${ }^{51}$ V. Kumar, ${ }^{51}$ A. K. Mohanty, ${ }^{51, \mathrm{c}}$ L. M. Pant, ${ }^{51}$ P. Shukla, ${ }^{51}$ A. Topkar, ${ }^{51}$ T. Aziz, ${ }^{52}$ S. Banerjee, ${ }^{52}$ S. Bhowmik, ${ }^{52 \text { aa }}$ R. M. Chatterjee, ${ }^{52}$ R. K. Dewanjee, ${ }^{52}$ S. Dugad, ${ }^{52}$ S. Ganguly, ${ }^{52}$ S. Ghosh, ${ }^{52}$ M. Guchait, ${ }^{52}$ A. Gurtu ${ }^{52, b b}$ G. Kole, ${ }^{52}$ S. Kumar,${ }^{52}$ B. Mahakud, ${ }^{52}$ M. Maity, ${ }^{52, \text { aa }}$ G. Majumder, ${ }^{52}$

K. Mazumdar, ${ }^{52}$ S. Mitra, ${ }^{52}$ G. B. Mohanty ${ }^{52}$ B. Parida, ${ }^{52}$ T. Sarkar, ${ }^{52, a a}$ N. Sur, ${ }^{52}$ B. Sutar,${ }^{52}$ N. Wickramage,,${ }^{52, c c}$

S. Chauhan, ${ }^{53}$ S. Dube ${ }^{53}$ A. Kapoor ${ }^{53}$ K. Kothekar ${ }^{53}$ S. Sharma, ${ }^{53}$ H. Bakhshiansohi, ${ }^{54}$ H. Behnamian, ${ }^{54}$

S. M. Etesami, ${ }^{54, d d}$ A. Fahim,,${ }^{54, e e}$ R. Goldouzian, ${ }^{54}$ M. Khakzad, ${ }^{54}$ M. Mohammadi Najafabadi, ${ }^{54}$ M. Naseri, ${ }^{54}$ S. Paktinat Mehdiabadi ${ }^{54}$ F. Rezaei Hosseinabadi, ${ }^{54}$ B. Safarzadeh, ${ }^{54, f f}$ M. Zeinali, ${ }^{54}$ M. Felcini, ${ }^{55}$ M. Grunewald, ${ }^{55}$

M. Abbrescia, ${ }^{56,56 \mathrm{~b}}$ C. Calabria, ${ }^{56 \mathrm{a}, 56 \mathrm{~b}}$ C. Caputo, ${ }^{56 \mathrm{a}, 56 \mathrm{~b}}$ A. Colaleo, ${ }^{56 \mathrm{a}}$ D. Creanza, ${ }^{56,56 \mathrm{c}}$ L. Cristella, ${ }^{56 a, 56 \mathrm{~b}}$ N. De Filippis ${ }^{56 a, 56 c}$ M. De Palma ${ }^{56 a, 56 b}$ L. Fiore,${ }^{56 a}$ G. Iaselli, ${ }^{56,56 c}$ G. Maggi,${ }^{56,56 c}$ M. Maggi, ${ }^{56 a}$ G. Miniello, ${ }^{56 a, 56 b}$ S. My, ${ }^{56,56 \mathrm{c}}$ S. Nuzzo, ${ }^{56 a, 56 \mathrm{~b}}$ A. Pompili, ${ }^{56 \mathrm{a}, 56 \mathrm{~b}}$ G. Pugliese,${ }^{56,56 \mathrm{c}}$ R. Radogna,${ }^{56,56 \mathrm{~b}}$ A. Ranieri, ${ }^{56 \mathrm{a}}$ G. Selvaggi, ${ }^{56,56 \mathrm{~b}}$ L. Silvestris, ${ }^{56 a, c}$ R. Venditti, ${ }^{56 a, 56 b}$ P. Verwilligen, ${ }^{56 a}$ G. Abbiendi, ${ }^{57 a}$ C. Battilana, ${ }^{57 a, c}$ A. C. Benvenuti, ${ }^{57 a}$

D. Bonacorsi,${ }^{57 a, 57 b}$ S. Braibant-Giacomelli, ${ }^{57 a, 57 b}$ L. Brigliadori, ${ }^{57 a, 57 b}$ R. Campanini, ${ }^{57 a, 57 b}$ P. Capiluppi, ${ }^{57 a, 57 b}$ A. Castro, ${ }^{57,57 b}$ F. R. Cavallo, ${ }^{57 a}$ S. S. Chhibra, ${ }^{57,57 b}$ G. Codispoti, ${ }^{57,57 b}$ M. Cuffiani, ${ }^{57 a, 57 b}$ G. M. Dallavalle, ${ }^{57 a}$ F. Fabbri, ${ }^{57 a}$ A. Fanfani, ${ }^{57 a, 57 b}$ D. Fasanella, ${ }^{57 a, 57 b}$ P. Giacomelli, ${ }^{57 a}$ C. Grandi, ${ }^{57 a}$ L. Guiducci, ${ }^{57 a, 57 b}$ S. Marcellini, ${ }^{57 a}$ G. Masetti,,${ }^{57 a}$ A. Montanari, ${ }^{57 \mathrm{a}}$ F. L. Navarria, ${ }^{57 \mathrm{a}, 57 \mathrm{~b}}$ A. Perrotta, ${ }^{57 \mathrm{a}}$ A. M. Rossi, ${ }^{57 a, 57 b}$ T. Rovelli, ${ }^{57 a, 57 b}$ G. P. Siroli, ${ }^{57 a, 57 b}$ N. Tosi, ${ }^{57 a, 57 b, c}$ R. Travaglini, ${ }^{57 a, 57 b}$ G. Cappello, ${ }^{58 \mathrm{a}}$ M. Chiorboli, ${ }^{58 \mathrm{a}, 58 \mathrm{~b}}$ S. Costa ${ }^{58 \mathrm{a}, 58 \mathrm{~b}}$ A. Di Mattia, ${ }^{58 \mathrm{a}}$ F. Giordano, ${ }^{58 \mathrm{a}, 58 \mathrm{~b}}$ R. Potenza, ${ }^{58 \mathrm{a}, 58 \mathrm{~b}}$ A. Tricomi ${ }^{58 a, 58 b}$ C. Tuve,${ }^{58 a, 58 b}$ G. Barbagli, ${ }^{59 a}$ V. Ciulli,${ }^{59 a, 59 b}$ C. Civinini,${ }^{59 a}$ R. D'Alessandro, ${ }^{59 a, 59 b}$ E. Focardi, ${ }^{59 a, 59 b}$ V. Gori ${ }^{59 a, 59 b}$ P. Lenzi,${ }^{59 a, 59 b}$ M. Meschini,${ }^{59 a}$ S. Paoletti, ${ }^{59 a}$ G. Sguazzoni, ${ }^{59 a}$ L. Viliani,${ }^{59,59 b, c}$ L. Benussi,${ }^{60}$ S. Bianco, ${ }^{60}$ 
F. Fabbri, ${ }^{60}$ D. Piccolo, ${ }^{60}$ F. Primavera, ${ }^{60, \mathrm{c}}$ V. Calvelli, ${ }^{61 \mathrm{a}, 61 \mathrm{~b}}$ F. Ferro, ${ }^{61 \mathrm{a}}$ M. Lo Vetere ${ }^{61 \mathrm{a}, 61 \mathrm{~b}}$ M. R. Monge,${ }^{61 \mathrm{a}, 61 \mathrm{~b}}$ E. Robutti, ${ }^{61 a}$ S. Tosi,${ }^{61 a, 61 b}$ L. Brianza, ${ }^{62 a}$ M. E. Dinardo, ${ }^{62 a, 62 b}$ S. Fiorendi ${ }^{62 a, 62 b}$ S. Gennai, ${ }^{62 a}$ R. Gerosa, ${ }^{62 a, 62 b}$ A. Ghezzi ${ }^{62 a, 62 b}$ P. Govoni, ${ }^{62 a, 62 b}$ S. Malvezzi, ${ }^{62 a}$ R. A. Manzoni, ${ }^{62 a, 62 b, c}$ B. Marzocchi, ${ }^{62 a, 62 b}$ D. Menasce, ${ }^{62 a}$ L. Moroni, ${ }^{62 a}$ M. Paganoni, ${ }^{62 a, 62 b}$ D. Pedrini, ${ }^{62 a}$ S. Ragazzi, ${ }^{62 a, 62 b}$ N. Redaelli, ${ }^{62 a}$ T. Tabarelli de Fatis, ${ }^{62 a, 62 b}$ S. Buontempo, ${ }^{63 a}$ N. Cavallo, ${ }^{63 a, 63 c}$ S. Di Guida, ${ }^{63 a, 63 d, c}$ M. Esposito, ${ }^{63 a, 63 b}$ F. Fabozzi, ${ }^{63 a, 63 c}$ A. O. M. Iorio, ${ }^{63 a, 63 b}$ G. Lanza, ${ }^{63 a}$ L. Lista, ${ }^{63 a}$ S. Meola ${ }^{63 a, 63 d, c}$ M. Merola,${ }^{63 a}$ P. Paolucci, ${ }^{63 a, c}$ C. Sciacca,${ }^{63 a, 63 b}$ F. Thyssen, ${ }^{63 a}$ P. Azzi ${ }^{64 a, c}$ N. Bacchetta, ${ }^{64 a}$ L. Benato,${ }^{64 a, 64 b}$ D. Bisello, ${ }^{64 a, 64 b}$ A. Boletti, ${ }^{64 a, 64 b}$ A. Branca, ${ }^{64 a, 64 b}$ R. Carlin,${ }^{64 a, 64 b}$ P. Checchia, ${ }^{64 a}$ M. Dall'Osso, ${ }^{64 a, 64 b, c}$ T. Dorigo, ${ }^{64 a}$

U. Dosselli, ${ }^{64 a}$ F. Gasparini, ${ }^{64 a, 64 b}$ U. Gasparini, ${ }^{64 a, 64 b}$ A. Gozzelino, ${ }^{64 a}$ K. Kanishchev, ${ }^{64 a, 64 c}$ S. Lacaprara, ${ }^{64 a}$ M. Margoni, ${ }^{64 a, 64 b}$ A. T. Meneguzzo, ${ }^{64 a, 64 b}$ J. Pazzini, ${ }^{64 a, 64 b, c}$ M. Pegoraro ${ }^{64 a}$ N. Pozzobon, ${ }^{64 a, 64 b}$ P. Ronchese, ${ }^{64 a, 64 b}$ F. Simonetto, ${ }^{64 a, 64 b}$ E. Torassa ${ }^{64 a}$ M. Tosi ${ }^{64 a, 64 b}$ M. Zanetti, ${ }^{64 a}$ P. Zotto, ${ }^{64 a, 64 b}$ A. Zucchetta ${ }^{64 a, 64 b, c}$ G. Zumerle, ${ }^{64 a, 64 b}$ A. Braghieri, ${ }^{65 a}$ A. Magnani, ${ }^{65 a}$ P. Montagna, ${ }^{65 a, 65 b}$ S. P. Ratti, ${ }^{65 a, 65 b}$ V. Re, ${ }^{65 a}$ C. Riccardi,${ }^{65 a, 65 b}$ P. Salvini, ${ }^{65 a}$ I. Vai, ${ }^{65 a}$

P. Vitulo, ${ }^{65 a, 65 b}$ L. Alunni Solestizi, ${ }^{66 a, 66 b}$ G. M. Bilei, ${ }^{66 \mathrm{a}}$ D. Ciangottini, ${ }^{66 a, 66 b, c}$ L. Fanò, ${ }^{66 a, 66 b}$ P. Lariccia, ${ }^{66 a, 66 b}$ G. Mantovani, ${ }^{66 a, 66 b}$ M. Menichelli, ${ }^{66 a}$ A. Saha, ${ }^{66 a}$ A. Santocchia,${ }^{66,66 b}$ K. Androsov, ${ }^{67 a, g g}$ P. Azzurri, ${ }^{67 a c c}$ G. Bagliesi, ${ }^{67 a}$ J. Bernardini, ${ }^{67 \mathrm{a}}$ T. Boccali, ${ }^{67 \mathrm{a}}$ R. Castaldi, ${ }^{67 \mathrm{a}}$ M. A. Ciocci, ${ }^{67 \mathrm{a}, \mathrm{gg}}$ R. Dell'Orso, ${ }^{67 \mathrm{a}}$ S. Donato, ${ }^{67 \mathrm{a}, 67 \mathrm{c}, \mathrm{c}}$ G. Fedi, ${ }^{67 \mathrm{a}}$ L. Foà, ${ }^{67 \mathrm{a}, 67 \mathrm{c}, \mathrm{a}}$ A. Giassi ${ }^{67 \mathrm{a}}$ M. T. Grippo, ${ }^{67 \mathrm{a}, \mathrm{gg}}$ F. Ligabue ${ }^{67 \mathrm{a}, 67 \mathrm{c}}$ T. Lomtadze, ${ }^{67 \mathrm{a}}$ L. Martini, ${ }^{67 \mathrm{a}, 67 \mathrm{~b}}$ A. Messineo, ${ }^{67 a, 67 \mathrm{~b}}$ F. Palla, ${ }^{67 \mathrm{a}}$ A. Rizzi, ${ }^{67,67 b}$ A. Savoy-Navarro, ${ }^{67 a, h h}$ A. T. Serban, ${ }^{67 a}$ P. Spagnolo, ${ }^{67 a}$ R. Tenchini, ${ }^{67 a}$ G. Tonelli, ${ }^{67 a, 67 b}$ A. Venturi, ${ }^{67 a}$ P. G. Verdini ${ }^{67 a}$ L. Barone, ${ }^{68 a, 68 b}$ F. Cavallari, ${ }^{68 a}$ G. D'imperio, ${ }^{68 a, 68 b, c}$ D. Del Re ${ }^{68 a, 68 b, c}$ M. Diemoz, ${ }^{68 a}$ S. Gelli, ${ }^{68 a, 68 b}$ C. Jorda, ${ }^{68 \mathrm{a}}$ E. Longo, ${ }^{68 \mathrm{a}, 68 \mathrm{~b}}$ F. Margaroli, ${ }^{68 \mathrm{a}, 68 \mathrm{~b}}$ P. Meridiani, ${ }^{68 \mathrm{a}}$ G. Organtini, ${ }^{68 \mathrm{a}, 68 \mathrm{~b}}$ R. Paramatti, ${ }^{68 \mathrm{a}}$ F. Preiato, ${ }^{68 a, 68 b}$ S. Rahatlou ${ }^{68 \mathrm{a}, 68 \mathrm{~b}}$ C. Rovelli, ${ }^{68 \mathrm{a}}$ F. Santanastasio, ${ }^{6 \mathrm{a}, 68 \mathrm{~b}}$ P. Traczyk, ${ }^{68 \mathrm{a}, 68 \mathrm{~b}, \mathrm{c}}$ N. Amapane,${ }^{6 \mathrm{a}, 69 \mathrm{~b}}$ R. Arcidiacono, ${ }^{69 \mathrm{a}, 69 \mathrm{c}, \mathrm{c}}$ S. Argiro, ${ }^{69 a, 69 b}$ M. Arneodo, ${ }^{69 a, 69 c}$ R. Bellan, ${ }^{69 a, 69 b}$ C. Biino, ${ }^{69 a}$ N. Cartiglia,${ }^{69 a}$ M. Costa, ${ }^{69 a, 69 b}$ R. Covarelli, ${ }^{69 a, 69 b}$ A. Degano ${ }^{69 \mathrm{a}, 69 \mathrm{~b}}$ N. Demaria, ${ }^{69 \mathrm{a}}$ L. Finco,${ }^{69 \mathrm{a}, 69 \mathrm{~b}, \mathrm{c}}$ B. Kiani ${ }^{69 \mathrm{a}, 69 \mathrm{~b}}$ C. Mariotti,${ }^{69 \mathrm{a}}$ S. Maselli, ${ }^{69 \mathrm{a}}$ E. Migliore,${ }^{69 \mathrm{a}, 69 \mathrm{~b}}$ V. Monaco, ${ }^{69 a, 69 b}$ E. Monteil, ${ }^{69,69 b}$ M. M. Obertino, ${ }^{69 a, 69 b}$ L. Pacher,${ }^{69 a, 69 b}$ N. Pastrone, ${ }^{69 a}$ M. Pelliccioni, ${ }^{69 a}$ G. L. Pinna Angioni ${ }^{69 \mathrm{a}, 69 \mathrm{~b}}$ F. Ravera ${ }^{69 \mathrm{a}, 69 \mathrm{~b}}$ A. Romero, ${ }^{69 a, 69 \mathrm{~b}}$ M. Ruspa,${ }^{69 a, 69 \mathrm{c}}$ R. Sacchi, ${ }^{69 a, 69 \mathrm{~b}}$ A. Solano, ${ }^{69 \mathrm{a}, 69 \mathrm{~b}}$ A. Staiano, ${ }^{69 a}$ S. Belforte, ${ }^{70 a}$ V. Candelise,${ }^{70 a, 70 b}$ M. Casarsa, ${ }^{70 a}$ F. Cossutti, ${ }^{70 a}$ G. Della Ricca, ${ }^{70 a, 70 b}$ B. Gobbo, ${ }^{70 a}$ C. La Licata, ${ }^{70 a, 70 b}$ M. Marone, ${ }^{70 a, 70 b}$ A. Schizzi, ${ }^{70 a, 70 b}$ A. Zanetti, ${ }^{70 a}$ A. Kropivnitskaya, ${ }^{71}$ S. K. Nam, ${ }^{71}$ D. H. Kim, ${ }^{72}$ G. N. Kim, ${ }^{72}$ M. S. Kim, ${ }^{72}$ D. J. Kong, ${ }^{72}$ S. Lee, ${ }^{72}$ Y. D. Oh, ${ }^{72}$ A. Sakharov, ${ }^{72}$ D. C. Son, ${ }^{72}$ J. A. Brochero Cifuentes ${ }^{73}$ H. Kim, ${ }^{73}$ T. J. Kim, ${ }^{73}$ S. Song ${ }^{74}$ S. Choi, ${ }^{75}$ Y. Go, ${ }^{75}$ D. Gyun, ${ }^{75}$ B. Hong,${ }^{75}$ H. Kim, ${ }^{75}$ Y. Kim, ${ }^{75}$ B. Lee,${ }^{75}$ K. Lee, ${ }^{75}$ K. S. Lee, ${ }^{75}$ S. Lee, ${ }^{75}$ S. K. Park, ${ }^{75}$ Y. Roh,${ }^{75}$ H. D. Yoo ${ }^{76}$ M. Choi,${ }^{77}$ H. Kim,${ }^{77}$ J. H. Kim, ${ }^{77}$ J. S. H. Lee, ${ }^{77}$ I. C. Park, ${ }^{77}$ G. Ryu, ${ }^{77}$ M. S. Ryu,${ }^{77}$ Y. Choi, ${ }^{78}$ J. Goh, ${ }^{78}$ D. Kim, ${ }^{78}$ E. Kwon, ${ }^{78}$ J. Lee, ${ }^{78}$ I. Yu, ${ }^{78}$ V. Dudenas, ${ }^{79}$ A. Juodagalvis ${ }^{79}$ J. Vaitkus, ${ }^{79}$ I. Ahmed ${ }^{80}$ Z. A. Ibrahim, ${ }^{80}$ J. R. Komaragiri, ${ }^{80}$ M. A. B. Md Ali, ${ }^{80, i i}$ F. Mohamad Idris, ${ }^{80, j j}$ W. A. T. Wan Abdullah, ${ }^{80}$ M. N. Yusli, ${ }^{80}$ E. Casimiro Linares, ${ }^{81}$ H. Castilla-Valdez,${ }^{81}$ E. De La Cruz-Burelo, ${ }^{81}$ I. Heredia-De La Cruz,${ }^{81, k k}$ A. Hernandez-Almada, ${ }^{81}$ R. Lopez-Fernandez,${ }^{81}$ A. Sanchez-Hernandez, ${ }^{81}$ S. Carrillo Moreno, ${ }^{82}$ F. Vazquez Valencia, ${ }^{82}$ I. Pedraza ${ }^{83}$ H. A. Salazar Ibarguen, ${ }^{83}$ A. Morelos Pineda,${ }^{84}$ D. Krofcheck ${ }^{85}$ P. H. Butler, ${ }^{86}$ A. Ahmad ${ }^{87}$ M. Ahmad ${ }^{87}$ Q. Hassan, ${ }^{87}$ H. R. Hoorani, ${ }^{87}$ W. A. Khan, ${ }^{87}$ T. Khurshid ${ }^{87}$ M. Shoaib, ${ }^{87}$ H. Bialkowska, ${ }^{88}$ M. Bluj, ${ }^{88}$ B. Boimska, ${ }^{88}$ T. Frueboes, ${ }^{88}$ M. Górski, ${ }^{88}$ M. Kazana, ${ }^{88}$ K. Nawrocki, ${ }^{88}$ K. Romanowska-Rybinska, ${ }^{88}$ M. Szleper, ${ }^{88}$ P. Zalewski, ${ }^{88}$ G. Brona, ${ }^{89}$ K. Bunkowski, ${ }^{89}$ A. Byszuk,${ }^{89,11}$ K. Doroba, ${ }^{89}$ A. Kalinowski, ${ }^{89}$ M. Konecki, ${ }^{89}$ J. Krolikowski, ${ }^{89}$ M. Misiura ${ }^{89}$ M. Olszewski, ${ }^{89}$ M. Walczak ${ }^{89}$ P. Bargassa, ${ }^{90}$ C. Beirão Da Cruz E Silva, ${ }^{90}$ A. Di Francesco, ${ }^{90}$ P. Faccioli,${ }^{90}$ P. G. Ferreira Parracho, ${ }^{90}$ M. Gallinaro, ${ }^{90}$ N. Leonardo, ${ }^{90}$ L. Lloret Iglesias, ${ }^{90}$ F. Nguyen, ${ }^{90}$ J. Rodrigues Antunes,${ }^{90}$ J. Seixas, ${ }^{90}$ O. Toldaiev, ${ }^{90}$ D. Vadruccio,${ }^{90}$ J. Varela,${ }^{90}$ P. Vischia, ${ }^{90}$ S. Afanasiev, ${ }^{91}$ P. Bunin, ${ }^{91}$ M. Gavrilenko, ${ }^{91}$ I. Golutvin, ${ }^{91}$ I. Gorbunov, ${ }^{91}$ A. Kamenev, ${ }^{91}$ V. Karjavin, ${ }^{91}$ V. Konoplyanikov, ${ }^{91}$ A. Lanev, ${ }^{91}$ A. Malakhov, ${ }^{91}$ V. Matveev, ${ }^{91, \mathrm{~mm}, \mathrm{nn}}$ P. Moisenz, ${ }^{91}$ V. Palichik, ${ }^{91}$ V. Perelygin, ${ }^{91}$ S. Shmatov, ${ }^{91}$ S. Shulha, ${ }^{91}$ N. Skatchkov, ${ }^{91}$ V. Smirnov, ${ }^{91}$ A. Zarubin, ${ }^{91}$ V. Golovtsov, ${ }^{92}$ Y. Ivanov,${ }^{92}$ V. Kim,${ }^{92,00}$ E. Kuznetsovaa, ${ }^{92}$ P. Levchenko, ${ }^{92}$ V. Murzin, ${ }^{92}$ V. Oreshkin, ${ }^{92}$ I. Smirnov, ${ }^{92}$ V. Sulimov, ${ }^{92}$ L. Uvarov, ${ }^{92}$ S. Vavilov, ${ }^{92}$ A. Vorobyev, ${ }^{92}$ Yu. Andreev, ${ }^{93}$ A. Dermenev, ${ }^{93}$ S. Gninenko, ${ }^{93}$ N. Golubev, ${ }^{93}$ A. Karneyeu, ${ }^{93}$ M. Kirsanov, ${ }^{93}$ N. Krasnikov, ${ }^{93}$ A. Pashenkov, ${ }^{93}$ D. Tlisov, ${ }^{93}$ A. Toropin, ${ }^{93}$ V. Epshteyn, ${ }^{94}$ V. Gavrilov, ${ }^{94}$ N. Lychkovskaya,${ }^{94}$ V. Popov, ${ }^{94}$ I. Pozdnyakov, ${ }^{94}$ G. Safronov, ${ }^{94}$ A. Spiridonov, ${ }^{94}$ E. Vlasov, ${ }^{94}$ A. Zhokin, ${ }^{94}$ A. Bylinkin, ${ }^{95}$ V. Andreev,${ }^{96}$ M. Azarkin, ${ }^{96, n n}$ I. Dremin,${ }^{96, n n}$ M. Kirakosyan ${ }^{96}$ A. Leonidov, ${ }^{96, n n}$ G. Mesyats, ${ }^{96}$ S. V. Rusakov, ${ }^{96}$ A. Baskakov, ${ }^{97}$ A. Belyaev,${ }^{97}$ E. Boos, ${ }^{97}$ V. Bunichev, ${ }^{97}$ M. Dubinin,,${ }^{97, p p}$ L. Dudko, ${ }^{97}$ A. Gribushin,${ }^{97}$ V. Klyukhin, ${ }^{97}$ O. Kodolova, ${ }^{97}$ N. Korneeva,${ }^{97}$ I. Lokhtin, ${ }^{97}$ I. Myagkov, ${ }^{97}$ S. Obraztsov, ${ }^{97}$ M. Perfilov, ${ }^{97}$ V. Savrin, ${ }^{97}$ I. Azhgirey, ${ }^{98}$ I. Bayshev, ${ }^{98}$ S. Bitioukov, ${ }^{98}$ V. Kachanov, ${ }^{98}$ A. Kalinin, ${ }^{98}$ D. Konstantinov, ${ }^{98}$ 
V. Krychkine, ${ }^{98}$ V. Petrov, ${ }^{98}$ R. Ryutin, ${ }^{98}$ A. Sobol,${ }^{98}$ L. Tourtchanovitch, ${ }^{98}$ S. Troshin,${ }^{98}$ N. Tyurin, ${ }^{98}$ A. Uzunian, ${ }^{98}$ A. Volkov, ${ }^{98}$ P. Adzic, ${ }^{99, q 9}$ P. Cirkovic, ${ }^{99}$ J. Milosevic, ${ }^{99}$ V. Rekovic, ${ }^{99}$ J. Alcaraz Maestre, ${ }^{100}$ E. Calvo, ${ }^{100}$ M. Cerrada, ${ }^{100}$ M. Chamizo Llatas, ${ }^{100}$ N. Colino, ${ }^{100}$ B. De La Cruz, ${ }^{100}$ A. Delgado Peris, ${ }^{100}$ A. Escalante Del Valle,${ }^{100}$

C. Fernandez Bedoya,${ }^{100}$ J. P. Fernández Ramos, ${ }^{100}$ J. Flix, ${ }^{100}$ M. C. Fouz,${ }^{100}$ P. Garcia-Abia, ${ }^{100}$ O. Gonzalez Lopez, ${ }^{100}$ S. Goy Lopez,${ }^{100}$ J. M. Hernandez, ${ }^{100}$ M. I. Josa ${ }^{100}$ E. Navarro De Martino, ${ }^{100}$ A. Pérez-Calero Yzquierdo, ${ }^{100}$ J. Puerta Pelayo, ${ }^{100}$ A. Quintario Olmeda,${ }^{100}$ I. Redondo, ${ }^{100}$ L. Romero, ${ }^{100}$ J. Santaolalla, ${ }^{100}$ M. S. Soares, ${ }^{100}$ C. Albajar, ${ }^{101}$ J. F. de Trocóniz, ${ }^{101}$ M. Missiroli, ${ }^{101}$ D. Moran, ${ }^{101}$ J. Cuevas, ${ }^{102}$ J. Fernandez Menendez, ${ }^{102}$ S. Folgueras, ${ }^{102}$

I. Gonzalez Caballero, ${ }^{102}$ E. Palencia Cortezon, ${ }^{102}$ S. Sanchez Cruz, ${ }^{102}$ J. M. Vizan Garcia, ${ }^{102}$ I. J. Cabrillo, ${ }^{103}$ A. Calderon, ${ }^{103}$ J. R. Castiñeiras De Saa, ${ }^{103}$ P. De Castro Manzano, ${ }^{103}$ M. Fernandez,${ }^{103}$ J. Garcia-Ferrero, ${ }^{103}$ G. Gomez, ${ }^{103}$ A. Lopez Virto, ${ }^{103}$ J. Marco,${ }^{103}$ R. Marco, ${ }^{103}$ C. Martinez Rivero, ${ }^{103}$ F. Matorras, ${ }^{103}$ J. Piedra Gomez, ${ }^{103}$ T. Rodrigo, ${ }^{103}$ A. Y. Rodríguez-Marrero, ${ }^{103}$ A. Ruiz-Jimeno, ${ }^{103}$ L. Scodellaro,${ }^{103}$ N. Trevisani, ${ }^{103}$ I. Vila, ${ }^{103}$ R. Vilar Cortabitarte,${ }^{103}$ D. Abbaneo,${ }^{104}$ E. Auffray, ${ }^{104}$ G. Auzinger, ${ }^{104}$ M. Bachtis, ${ }^{104}$ P. Baillon, ${ }^{104}$ A. H. Ball, ${ }^{104}$ D. Barney,${ }^{104}$ A. Benaglia, ${ }^{104}$ J. Bendavid, ${ }^{104}$ L. Benhabib, ${ }^{104}$ J. F. Benitez, ${ }^{104}$ G. M. Berruti, ${ }^{104}$ P. Bloch, ${ }^{104}$ A. Bocci, ${ }^{104}$ A. Bonato, ${ }^{104}$ C. Botta, ${ }^{104}$ H. Breuker, ${ }^{104}$ T. Camporesi, ${ }^{104}$ R. Castello, ${ }^{104}$ G. Cerminara, ${ }^{104}$ M. D'Alfonso, ${ }^{104}$ D. d'Enterria, ${ }^{104}$ A. Dabrowski, ${ }^{104}$ V. Daponte, ${ }^{104}$ A. David, ${ }^{104}$ M. De Gruttola, ${ }^{104}$ F. De Guio, ${ }^{104}$ A. De Roeck, ${ }^{104}$ S. De Visscher, ${ }^{104}$ E. Di Marco, ${ }^{104, \text { rr }}$ M. Dobson, ${ }^{104}$ M. Dordevic, ${ }^{104}$ B. Dorney, ${ }^{104}$ T. du Pree, ${ }^{104}$ D. Duggan, ${ }^{104}$ M. Dünser, ${ }^{104}$ N. Dupont, ${ }^{104}$ A. Elliott-Peisert, ${ }^{104}$ G. Franzoni, ${ }^{104}$ J. Fulcher, ${ }^{104}$ W. Funk, ${ }^{104}$ D. Gigi,${ }^{104}$ K. Gill, ${ }^{104}$ D. Giordano, ${ }^{104}$ M. Girone, ${ }^{104}$ F. Glege,${ }^{104}$ R. Guida, ${ }^{104}$ S. Gundacker, ${ }^{104}$ M. Guthoff, ${ }^{104}$ J. Hammer, ${ }^{104}$ P. Harris, ${ }^{104}$ J. Hegeman, ${ }^{104}$ V. Innocente, ${ }^{104}$ P. Janot,${ }^{104}$ H. Kirschenmann, ${ }^{104}$ M. J. Kortelainen, ${ }^{104}$ K. Kousouris, ${ }^{104}$ K. Krajczar, ${ }^{104}$ P. Lecoq,${ }^{104}$ C. Lourenço, ${ }^{104}$ M. T. Lucchini, ${ }^{104}$ N. Magini, ${ }^{104}$ L. Malgeri, ${ }^{104}$ M. Mannelli, ${ }^{104}$ A. Martelli, ${ }^{104}$ L. Masetti, ${ }^{104}$ F. Meijers, ${ }^{104}$ S. Mersi,${ }^{104}$ E. Meschi, ${ }^{104}$ F. Moortgat,${ }^{104}$ S. Morovic, ${ }^{104}$ M. Mulders, ${ }^{104}$ M. V. Nemallapudi, ${ }^{104}$ H. Neugebauer, ${ }^{104}$ S. Orfanelli, ${ }^{104, s s}$ L. Orsini, ${ }^{104}$ L. Pape, ${ }^{104}$ E. Perez, ${ }^{104}$ M. Peruzzi, ${ }^{104}$ A. Petrilli, ${ }^{104}$ G. Petrucciani, ${ }^{104}$ A. Pfeiffer, ${ }^{104}$ D. Piparo, ${ }^{104}$ A. Racz, ${ }^{104}$ T. Reis, ${ }^{104}$ G. Rolandi, ${ }^{104, t t}$ M. Rovere, ${ }^{104}$ M. Ruan, ${ }^{104}$ H. Sakulin, ${ }^{104}$ C. Schäfer,${ }^{104}$ C. Schwick,${ }^{104}$ M. Seidel, ${ }^{104}$ A. Sharma,${ }^{104}$ P. Silva, ${ }^{104}$ M. Simon, ${ }^{104}$ P. Sphicas, ${ }^{104, \text { uu }}$ J. Steggemann, ${ }^{104}$ B. Stieger,${ }^{104}$ M. Stoye,${ }^{104}$ Y. Takahashi, ${ }^{104}$ D. Treille, ${ }^{104}$ A. Triossi,${ }^{104}$ A. Tsirou, ${ }^{104}$ G. I. Veres, ${ }^{104, x}$ N. Wardle, ${ }^{104}$ H. K. Wöhri, ${ }^{104}$ A. Zagozdzinska, ${ }^{104,11}$ W. D. Zeuner, ${ }^{104}$ W. Bertl, ${ }^{105}$ K. Deiters, ${ }^{105}$ W. Erdmann, ${ }^{105}$ R. Horisberger, ${ }^{105}$ Q. Ingram, ${ }^{105}$ H. C. Kaestli, ${ }^{105}$ D. Kotlinski, ${ }^{105}$ U. Langenegger, ${ }^{105}$ D. Renker, ${ }^{105}$ T. Rohe, ${ }^{105}$ F. Bachmair, ${ }^{106}$ L. Bäni, ${ }^{106}$ L. Bianchini, ${ }^{106}$ B. Casal,,${ }^{106}$ G. Dissertori, ${ }^{106}$ M. Dittmar, ${ }^{106}$ M. Donegà, ${ }^{106}$ P. Eller, ${ }^{106}$ C. Grab,${ }^{106}$ C. Heidegger, ${ }^{106}$ D. Hits, ${ }^{106}$ J. Hoss, ${ }^{106}$ G. Kasieczka, ${ }^{106}$ W. Lustermann, ${ }^{106}$ B. Mangano, ${ }^{106}$ M. Marionneau, ${ }^{106}$ P. Martinez Ruiz del Arbol, ${ }^{106}$ M. Masciovecchio, ${ }^{106}$ D. Meister, ${ }^{106}$ F. Micheli, ${ }^{106}$ P. Musella, ${ }^{106}$ F. Nessi-Tedaldi, ${ }^{106}$ F. Pandolfi, ${ }^{106}$ J. Pata, ${ }^{106}$ F. Pauss, ${ }^{106}$ L. Perrozzi, ${ }^{106}$ M. Quittnat, ${ }^{106}$ M. Rossini, ${ }^{106}$ A. Starodumov, ${ }^{106, v v}$ M. Takahashi, ${ }^{106}$ V. R. Tavolaro, ${ }^{106} \mathrm{~K}$. Theofilatos, ${ }^{106}$ R. Wallny, ${ }^{106}$ T. K. Aarrestad, ${ }^{107}$ C. Amsler, ${ }^{107, w w}$ L. Caminada, ${ }^{107}$ M. F. Canelli, ${ }^{107}$ V. Chiochia, ${ }^{107}$ A. De Cosa, ${ }^{107}$ C. Galloni, ${ }^{107}$ A. Hinzmann, ${ }^{107}$

T. Hreus, ${ }^{107}$ B. Kilminster, ${ }^{107}$ C. Lange, ${ }^{107}$ J. Ngadiuba, ${ }^{107}$ D. Pinna, ${ }^{107}$ G. Rauco, ${ }^{107}$ P. Robmann, ${ }^{107}$ F. J. Ronga, ${ }^{107}$ D. Salerno, ${ }^{107}$ Y. Yang, ${ }^{107}$ M. Cardaci, ${ }^{108}$ K. H. Chen, ${ }^{108}$ T. H. Doan, ${ }^{108}$ Sh. Jain, ${ }^{108}$ R. Khurana, ${ }^{108}$ M. Konyushikhin, ${ }^{108}$ C. M. Kuo, ${ }^{108}$ W. Lin, ${ }^{108}$ Y. J. Lu, ${ }^{108}$ A. Pozdnyakov, ${ }^{108}$ S. S. Yu, ${ }^{108}$ Arun Kumar, ${ }^{109}$ R. Bartek, ${ }^{109}$ P. Chang, ${ }^{109}$ Y. H. Chang, ${ }^{109}$ Y. W. Chang, ${ }^{109}$ Y. Chao, ${ }^{109}$ K. F. Chen, ${ }^{109}$ P. H. Chen, ${ }^{109}$ C. Dietz, ${ }^{109}$ F. Fiori, ${ }^{109}$ U. Grundler, ${ }^{109}$ W.-S. Hou, ${ }^{109}$ Y. Hsiung, ${ }^{109}$ Y. F. Liu, ${ }^{109}$ R.-S. Lu, ${ }^{109}$ M. Miñano Moya, ${ }^{109}$ E. Petrakou, ${ }^{109}$ J. f. Tsai, ${ }^{109}$ Y. M. Tzeng, ${ }^{109}$ B. Asavapibhop, ${ }^{110}$ K. Kovitanggoon, ${ }^{110}$ G. Singh,${ }^{110}$ N. Srimanobhas, ${ }^{110}$ N. Suwonjandee, ${ }^{110}$ A. Adiguzel,,${ }^{11}$ M. N. Bakirci, ${ }^{11, x x}$ S. Cerci, ${ }^{111, y y}$ Z. S. Demiroglu, ${ }^{111}$ C. Dozen, ${ }^{111}$ I. Dumanoglu, ${ }^{111}$ E. Eskut, ${ }^{111}$ F. H. Gecit, ${ }^{111}$ S. Girgis, ${ }^{111}$ G. Gokbulut, ${ }^{111}$ Y. Guler, ${ }^{111}$ E. Gurpinar, ${ }^{111}$ I. Hos, ${ }^{111}$ E. E. Kangal, ${ }^{111, z z}$ G. Onengut, ${ }^{11, \text { aaa }}$ M. Ozcan, ${ }^{111}$ K. Ozdemir, ${ }^{111, b b b}$ A. Polatoz, ${ }^{111}$ D. Sunar Cerci, ${ }^{111, y y}$ M. Vergili, ${ }^{111}$ C. Zorbilmez, ${ }^{111}$ I. V. Akin, ${ }^{112}$ B. Bilin, ${ }^{112}$ S. Bilmis, ${ }^{112}$ B. Isildak, ${ }^{12, \text { ccc }}$ G. Karapinar, ${ }^{112, \text { ddd }}$ M. Yalvac, ${ }^{112}$ M. Zeyrek, ${ }^{112}$ E. Gülmez, ${ }^{113}$ M. Kaya, ${ }^{113, \text { eee }}$ O. Kaya, ${ }^{113, f f f}$ E. A. Yetkin, ${ }^{113, \text { ggg }}$

T. Yetkin, ${ }^{113, \text { hhh }}$ A. Cakir, ${ }^{114}$ K. Cankocak, ${ }^{114}$ S. Sen, ${ }^{114, \text { iii }}$ F. I. Vardarll,${ }^{114}$ B. Grynyov, ${ }^{115}$ L. Levchuk, ${ }^{116}$ P. Sorokin,${ }^{116}$ R. Aggleton, ${ }^{117}$ F. Ball, ${ }^{117}$ L. Beck, ${ }^{117}$ J. J. Brooke, ${ }^{117}$ E. Clement, ${ }^{117}$ D. Cussans, ${ }^{117}$ H. Flacher,${ }^{117}$ J. Goldstein, ${ }^{117}$ M. Grimes, ${ }^{117}$ G. P. Heath, ${ }^{117}$ H. F. Heath, ${ }^{117}$ J. Jacob, ${ }^{117}$ L. Kreczko, ${ }^{117}$ C. Lucas, ${ }^{117}$ Z. Meng, ${ }^{117}$ D. M. Newbold, ${ }^{117, j j j}$ S. Paramesvaran, ${ }^{117}$ A. Poll, ${ }^{117}$ T. Sakuma, ${ }^{117}$ S. Seif El Nasr-storey, ${ }^{117}$ S. Senkin, ${ }^{117}$ D. Smith, ${ }^{117}$ V. J. Smith, ${ }^{117}$ K. W. Bell, ${ }^{118}$ A. Belyaev, ${ }^{118, k k k}$ C. Brew, ${ }^{118}$ R. M. Brown, ${ }^{118}$ L. Calligaris, ${ }^{118}$ D. Cieri, ${ }^{118}$ D. J. A. Cockerill,,${ }^{118}$

J. A. Coughlan, ${ }^{118}$ K. Harder, ${ }^{118}$ S. Harper, ${ }^{118}$ E. Olaiya, ${ }^{118}$ D. Petyt, ${ }^{118}$ C. H. Shepherd-Themistocleous, ${ }^{118}$ A. Thea,${ }^{118}$ I. R. Tomalin, ${ }^{118}$ T. Williams, ${ }^{118}$ S. D. Worm, ${ }^{118}$ M. Baber,${ }^{119}$ R. Bainbridge, ${ }^{119}$ O. Buchmuller, ${ }^{119}$ A. Bundock, ${ }^{119}$ 
D. Burton, ${ }^{119}$ S. Casasso, ${ }^{119}$ M. Citron, ${ }^{119}$ D. Colling, ${ }^{119}$ L. Corpe,${ }^{119}$ N. Cripps,${ }^{119}$ P. Dauncey, ${ }^{119}$ G. Davies, ${ }^{119}$ A. De Wit ${ }^{119}$ M. Della Negra, ${ }^{119}$ P. Dunne, ${ }^{119}$ A. Elwood, ${ }^{119}$ W. Ferguson, ${ }^{119}$ D. Futyan, ${ }^{119}$ G. Hall, ${ }^{119}$ G. Iles,${ }^{119}$ M. Kenzie, ${ }^{119}$ R. Lane, ${ }^{119}$ R. Lucas, ${ }^{119, j j}$ L. Lyons, ${ }^{119}$ A.-M. Magnan, ${ }^{119}$ S. Malik, ${ }^{119}$ J. Nash, ${ }^{119}$ A. Nikitenko, ${ }^{119, \mathrm{vv}}$ J. Pela ${ }^{119}$ M. Pesaresi, ${ }^{119}$ K. Petridis,${ }^{119}$ D. M. Raymond, ${ }^{119}$ A. Richards,${ }^{119}$ A. Rose, ${ }^{119}$ C. Seez, ${ }^{119}$ A. Tapper, ${ }^{119}$ K. Uchida, ${ }^{119}$ M. Vazquez Acosta, ${ }^{119,111}$ T. Virdee, ${ }^{119}$ S. C. Zenz, ${ }^{119}$ J. E. Cole,${ }^{120}$ P. R. Hobson, ${ }^{120}$ A. Khan, ${ }^{120}$ P. Kyberd, ${ }^{120}$ D. Leggat, ${ }^{120}$ D. Leslie, ${ }^{120}$ I. D. Reid, ${ }^{120}$ P. Symonds, ${ }^{120}$ L. Teodorescu, ${ }^{120}$ M. Turner, ${ }^{120}$ A. Borzou, ${ }^{121}$ K. Call, ${ }^{121}$ J. Dittmann, ${ }^{121}$ K. Hatakeyama, ${ }^{121}$ H. Liu ${ }^{121}$ N. Pastika, ${ }^{121}$ O. Charaf, ${ }^{122}$ S. I. Cooper, ${ }^{122}$ C. Henderson, ${ }^{122}$ P. Rumerio, ${ }^{122}$ D. Arcaro, ${ }^{123}$ A. Avetisyan, ${ }^{123}$ T. Bose, ${ }^{123}$ C. Fantasia, ${ }^{123}$ D. Gastler, ${ }^{123}$ P. Lawson, ${ }^{123}$ D. Rankin, ${ }^{123}$ C. Richardson, ${ }^{123}$ J. Rohlf, ${ }^{123}$ J. St. John, ${ }^{123}$ L. Sulak, ${ }^{123}$ D. Zou, ${ }^{123}$ J. Alimena, ${ }^{124}$ E. Berry, ${ }^{124}$ S. Bhattacharya, ${ }^{124}$ D. Cutts, ${ }^{124}$ A. Ferapontov, ${ }^{124}$ A. Garabedian, ${ }^{124}$ J. Hakala, ${ }^{124}$ U. Heintz, ${ }^{124}$ E. Laird, ${ }^{124}$ G. Landsberg, ${ }^{124}$ Z. Mao, ${ }^{124}$ M. Narain, ${ }^{124}$ S. Piperov, ${ }^{124}$ S. Sagir, ${ }^{124}$ R. Syarif, ${ }^{124}$ R. Breedon, ${ }^{125}$ G. Breto, ${ }^{125}$ M. Calderon De La Barca Sanchez, ${ }^{125}$ S. Chauhan, ${ }^{125}$ M. Chertok, ${ }^{125}$ J. Conway, ${ }^{125}$ R. Conway, ${ }^{125}$ P. T. Cox,${ }^{125}$ R. Erbacher,${ }^{125}$ G. Funk, ${ }^{125}$ M. Gardner, ${ }^{125}$ W. Ko, ${ }^{125}$ R. Lander, ${ }^{125}$ C. Mclean, ${ }^{125}$ M. Mulhearn, ${ }^{125}$ D. Pellett, ${ }^{125}$ J. Pilot, ${ }^{125}$ F. Ricci-Tam, ${ }^{125}$ S. Shalhout, ${ }^{125}$ J. Smith, ${ }^{125}$ M. Squires, ${ }^{125}$ D. Stolp, ${ }^{125}$ M. Tripathi, ${ }^{125}$ S. Wilbur, ${ }^{125}$ R. Yohay, ${ }^{125}$ C. Bravo, ${ }^{126}$ R. Cousins,${ }^{126}$ P. Everaerts, ${ }^{126}$ A. Florent, ${ }^{126}$ J. Hauser, ${ }^{126}$ M. Ignatenko, ${ }^{126}$ D. Saltzberg, ${ }^{126}$ C. Schnaible, ${ }^{126}$ E. Takasugi, ${ }^{126}$ V. Valuev, ${ }^{126}$ M. Weber, ${ }^{126}$ K. Burt, ${ }^{127}$ R. Clare, ${ }^{127}$ J. Ellison, ${ }^{127}$ J. W. Gary, ${ }^{127}$ G. Hanson, ${ }^{127}$ J. Heilman, ${ }^{127}$ M. Ivova PANEVA, ${ }^{127}$ P. Jandir, ${ }^{127}$ E. Kennedy, ${ }^{127}$ F. Lacroix, ${ }^{127}$ O. R. Long, ${ }^{127}$ A. Luthra, ${ }^{127}$ M. Malberti, ${ }^{127}$ M. Olmedo Negrete, ${ }^{127}$ A. Shrinivas, ${ }^{127}$ H. Wei, ${ }^{127}$ S. Wimpenny, ${ }^{127}$ B. R. Yates, ${ }^{127}$ J. G. Branson, ${ }^{128}$ G. B. Cerati, ${ }^{128}$ S. Cittolin, ${ }^{128}$ R. T. D’Agnolo, ${ }^{128}$ M. Derdzinski, ${ }^{128}$ A. Holzner, ${ }^{128}$ R. Kelley, ${ }^{128}$ D. Klein, ${ }^{128}$ J. Letts, ${ }^{128}$ I. Macneill, ${ }^{128}$ D. Olivito, ${ }^{128}$ S. Padhi, ${ }^{128}$ M. Pieri, ${ }^{128}$ M. Sani, ${ }^{128}$ V. Sharma, ${ }^{128}$ S. Simon, ${ }^{128}$ M. Tadel, ${ }^{128}$ A. Vartak, ${ }^{128}$ S. Wasserbaech, ${ }^{128, m m m}$ C. Welke, ${ }^{128}$ F. Würthwein, ${ }^{128}$ A. Yagil, ${ }^{128}$ G. Zevi Della Porta, ${ }^{128}$ J. Bradmiller-Feld, ${ }^{129}$ C. Campagnari, ${ }^{129}$ A. Dishaw, ${ }^{129}$ V. Dutta, ${ }^{129}$ K. Flowers, ${ }^{129}$ M. Franco Sevilla, ${ }^{129}$ P. Geffert, ${ }^{129}$ C. George, ${ }^{129}$ F. Golf, ${ }^{129}$ L. Gouskos, ${ }^{129}$ J. Gran, ${ }^{129}$ J. Incandela, ${ }^{129}$ N. Mccoll, ${ }^{129}$ S. D. Mullin, ${ }^{129}$ J. Richman, ${ }^{129}$ D. Stuart, ${ }^{129}$ I. Suarez,${ }^{129}$ C. West,${ }^{129}$ J. Yoo, ${ }^{129}$ D. Anderson, ${ }^{130}$ A. Apresyan, ${ }^{130}$ A. Bornheim, ${ }^{130}$ J. Bunn, ${ }^{130}$ Y. Chen, ${ }^{130}$ J. Duarte ${ }^{130}$ A. Mott, ${ }^{130}$ H. B. Newman, ${ }^{130}$ C. Pena,${ }^{130}$ M. Pierini, ${ }^{130}$ M. Spiropulu, ${ }^{130}$ J. R. Vlimant, ${ }^{130}$ S. Xie,${ }^{130}$ R. Y. Zhu, ${ }^{130}$ M. B. Andrews, ${ }^{131}$ V. Azzolini, ${ }^{131}$ A. Calamba, ${ }^{131}$ B. Carlson, ${ }^{131}$ T. Ferguson, ${ }^{131}$ M. Paulini, ${ }^{131}$ J. Russ, ${ }^{131}$ M. Sun, ${ }^{131}$ H. Vogel, ${ }^{131}$ I. Vorobiev, ${ }^{131}$ J. P. Cumalat, ${ }^{132}$ W. T. Ford,${ }^{132}$ A. Gaz, ${ }^{132}$ F. Jensen, ${ }^{132}$ A. Johnson, ${ }^{132}$ M. Krohn, ${ }^{132}$ T. Mulholland, ${ }^{132}$ U. Nauenberg, ${ }^{132}$ K. Stenson, ${ }^{132}$ S. R. Wagner ${ }^{132}$ J. Alexander, ${ }^{133}$ A. Chatterjee, ${ }^{133}$ J. Chaves, ${ }^{133}$ J. Chu, ${ }^{133}$ S. Dittmer, ${ }^{133}$ N. Eggert, ${ }^{133}$ N. Mirman, ${ }^{133}$ G. Nicolas Kaufman, ${ }^{133}$ J. R. Patterson, ${ }^{133}$ A. Rinkevicius, ${ }^{133}$ A. Ryd, ${ }^{133}$ L. Skinnari, ${ }^{133}$ L. Soffi, ${ }^{133}$ W. Sun, ${ }^{133}$ S. M. Tan, ${ }^{133}$ W. D. Teo, ${ }^{133}$ J. Thom, ${ }^{133}$ J. Thompson, ${ }^{133}$ J. Tucker, ${ }^{133}$ Y. Weng, ${ }^{133}$ P. Wittich, ${ }^{133}$ S. Abdullin, ${ }^{134}$ M. Albrow, ${ }^{134}$ G. Apollinari, ${ }^{134}$ S. Banerjee, ${ }^{134}$ L. A. T. Bauerdick, ${ }^{134}$ A. Beretvas, ${ }^{134}$ J. Berryhill, ${ }^{134}$ P. C. Bhat ${ }^{134}$ G. Bolla, ${ }^{134}$ K. Burkett, ${ }^{134}$ J. N. Butler, ${ }^{134}$ H. W. K. Cheung, ${ }^{134}$ F. Chlebana, ${ }^{134}$ S. Cihangir, ${ }^{134}$ V. D. Elvira, ${ }^{134}$ I. Fisk, ${ }^{134}$ J. Freeman, ${ }^{134}$ E. Gottschalk, ${ }^{134}$ L. Gray, ${ }^{134}$ D. Green, ${ }^{134}$ S. Grünendahl, ${ }^{134}$ O. Gutsche, ${ }^{134}$ J. Hanlon, ${ }^{134}$ D. Hare, ${ }^{134}$ R. M. Harris, ${ }^{134}$ S. Hasegawa, ${ }^{134}$ J. Hirschauer, ${ }^{134}$ Z. Hu, ${ }^{134}$ B. Jayatilaka, ${ }^{134}$ S. Jindariani, ${ }^{134}$ M. Johnson, ${ }^{134}$ U. Joshi, ${ }^{134}$ A. W. Jung, ${ }^{134}$ B. Klimaa ${ }^{134}$ B. Kreis,${ }^{134}$ S. Lammel, ${ }^{134}$ J. Linacre, ${ }^{134}$ D. Lincoln, ${ }^{134}$ R. Lipton, ${ }^{134}$ T. Liu, ${ }^{134}$ R. Lopes De Sá, ${ }^{134}$ J. Lykken, ${ }^{134}$ K. Maeshima, ${ }^{134}$ J. M. Marraffino, ${ }^{134}$ S. Maruyama, ${ }^{134}$ D. Mason, ${ }^{134}$ P. McBride, ${ }^{134}$ P. Merkel, ${ }^{134}$ K. Mishra, ${ }^{134}$ S. Mrenna,${ }^{134}$ S. Nahn, ${ }^{134}$ C. Newman-Holmes, ${ }^{134, a}$ V. O’Dell, ${ }^{134}$ K. Pedro, ${ }^{134}$ O. Prokofyev, ${ }^{134}$ G. Raknesss ${ }^{134}$ E. Sexton-Kennedy, ${ }^{134}$ A. Soha, ${ }^{134}$ W. J. Spalding, ${ }^{134}$ L. Spiegel, ${ }^{134}$ N. Strobbe, ${ }^{134}$ L. Taylor, ${ }^{134}$ S. Tkaczyk, ${ }^{134}$ N. V. Tran, ${ }^{134}$ L. Uplegger,,${ }^{134}$

E. W. Vaandering, ${ }^{134}$ C. Vernieri, ${ }^{134}$ M. Verzocchi, ${ }^{134}$ R. Vidal,${ }^{134}$ H. A. Weber, ${ }^{134}$ A. Whitbeck,,${ }^{134}$ D. Acosta, ${ }^{135}$ P. Avery, ${ }^{135}$ P. Bortignon, ${ }^{135}$ D. Bourilkov, ${ }^{135}$ A. Carnes, ${ }^{135}$ M. Carver, ${ }^{135}$ D. Curry, ${ }^{135}$ S. Das ${ }^{135}$ R. D. Field, ${ }^{135}$ I. K. Furic,${ }^{135}$ S. V. Gleyzer, ${ }^{135}$ J. Hugon, ${ }^{135}$ J. Konigsberg, ${ }^{135}$ A. Korytov, ${ }^{135}$ K. Kotov, ${ }^{135}$ J. F. Low, ${ }^{135}$ P. Ma,${ }^{135}$ K. Matchev, ${ }^{135}$ H. Mei, ${ }^{135}$ P. Milenovic, ${ }^{135, n n n}$ G. Mitselmakher, ${ }^{135}$ D. Rank, ${ }^{135}$ R. Rossin, ${ }^{135}$ L. Shchutska, ${ }^{135}$ M. Snowball, ${ }^{135}$ D. Sperka, ${ }^{135}$ N. Terentyev, ${ }^{135}$ L. Thomas, ${ }^{135}$ J. Wang, ${ }^{135}$ S. Wang, ${ }^{135}$ J. Yelton, ${ }^{135}$ S. Hewamanage, ${ }^{136}$ S. Linn, ${ }^{136}$ P. Markowitz, ${ }^{136}$ G. Martinez, ${ }^{136}$ J. L. Rodriguez, ${ }^{136}$ A. Ackert, ${ }^{137}$ J. R. Adams,${ }^{137}$ T. Adams, ${ }^{137}$ A. Askew, ${ }^{137}$ S. Bein, ${ }^{137}$ J. Bochenek, ${ }^{137}$ B. Diamond, ${ }^{137}$ J. Haas, ${ }^{137}$ S. Hagopian, ${ }^{137}$ V. Hagopian, ${ }^{137}$ K. F. Johnson, ${ }^{137}$ A. Khatiwada, ${ }^{137}$ H. Prosper, ${ }^{137}$ M. Weinberg, ${ }^{137}$ M. M. Baarmand, ${ }^{138}$ V. Bhopatkar, ${ }^{138}$ S. Colafranceschi, ${ }^{138,000}$ M. Hohlmann, ${ }^{138}$ H. Kalakhety, ${ }^{138}$ D. Noonan, ${ }^{138}$ T. Roy ${ }^{138}$ F. Yumiceva, ${ }^{138}$ M. R. Adams,${ }^{139}$ L. Apanasevich,${ }^{139}$ D. Berry, ${ }^{139}$ R. R. Betts, ${ }^{139}$ I. Bucinskaite, ${ }^{139}$ R. Cavanaugh, ${ }^{139}$ O. Evdokimov, ${ }^{139}$ L. Gauthier, ${ }^{139}$ C. E. Gerber, ${ }^{139}$ D. J. Hofman, ${ }^{139}$ P. Kurt, ${ }^{139}$ C. O’Brien, ${ }^{139}$ I. D. Sandoval Gonzalez, ${ }^{139}$ C. Silkworth, ${ }^{139}$ P. Turner, ${ }^{139}$ N. Varelas, ${ }^{139}$ Z. Wu, ${ }^{139}$ M. Zakaria, ${ }^{139}$ B. Bilki, ${ }^{140, p p p}$ 
W. Clarida, ${ }^{140}$ K. Dilsiz, ${ }^{140}$ S. Durgut, ${ }^{140}$ R. P. Gandrajula, ${ }^{140}$ M. Haytmyradov, ${ }^{140}$ V. Khristenko, ${ }^{140}$ J.-P. Merlo, ${ }^{140}$ H. Mermerkaya, ${ }^{140, q q 9}$ A. Mestvirishvili, ${ }^{140}$ A. Moeller, ${ }^{140}$ J. Nachtman, ${ }^{140}$ H. Ogul, ${ }^{140}$ Y. Onel, ${ }^{140}$ F. Ozok, ${ }^{140, r r}$ A. Penzo, ${ }^{140}$ C. Snyder, ${ }^{140}$ E. Tiras, ${ }^{140}$ J. Wetzel, ${ }^{140}$ K. Yi, ${ }^{140}$ I. Anderson, ${ }^{141}$ B. A. Barnett, ${ }^{141}$ B. Blumenfeld, ${ }^{141}$ N. Eminizer, ${ }^{141}$ D. Fehling, ${ }^{141}$ L. Feng, ${ }^{141}$ A. V. Gritsan, ${ }^{141}$ P. Maksimovic, ${ }^{141}$ C. Martin, ${ }^{141}$ M. Osherson, ${ }^{141}$ J. Roskes, ${ }^{141}$ A. Sady, ${ }_{141}$ U. Sarica, ${ }^{141}$ M. Swartz, ${ }^{141}$ M. Xiao, ${ }^{141}$ Y. Xin,${ }^{141}$ C. You, ${ }^{141}$ P. Baringer, ${ }^{142}$ A. Bean ${ }^{142}$ G. Benelli, ${ }^{142}$ C. Bruner, ${ }^{142}$ R. P. Kenny III, ${ }^{142}$ D. Majumder, ${ }^{142}$ M. Malek, ${ }^{142}$ M. Murray, ${ }^{142}$ S. Sanders, ${ }^{142}$ R. Stringer, ${ }^{142}$ Q. Wang, ${ }^{142}$ A. Ivanov, ${ }^{143}$ K. Kaadze, ${ }^{143}$ S. Khalil, ${ }^{143}$ M. Makouski, ${ }^{143}$ Y. Maravin, ${ }^{143}$ A. Mohammadi, ${ }^{143}$ L. K. Saini, ${ }^{143}$ N. Skhirtladze, ${ }^{143}$ S. Toda, ${ }^{143}$ D. Lange, ${ }^{144}$ F. Rebassoo, ${ }^{144}$ D. Wright, ${ }^{144}$ C. Anelli, ${ }^{145}$ A. Baden, ${ }^{145}$ O. Baron, ${ }^{145}$ A. Belloni, ${ }^{145}$ B. Calvert, ${ }^{145}$ S. C. Eno, ${ }^{145}$ C. Ferraioli, ${ }^{145}$ J. A. Gomez,${ }^{145}$ N. J. Hadley, ${ }^{145}$ S. Jabeen, ${ }^{145}$ R. G. Kellogg, ${ }^{145}$ T. Kolberg, ${ }^{145}$ J. Kunkle, ${ }^{145}$ Y. Lu, ${ }^{145}$ A. C. Mignerey, ${ }^{145}$ Y. H. Shin, ${ }^{145}$ A. Skuja, ${ }^{145}$ M. B. Tonjes, ${ }^{145}$ S. C. Tonwar, ${ }^{145}$ A. Apyan, ${ }^{146}$ R. Barbieri, ${ }^{146}$ A. Baty,${ }^{146}$ K. Bierwagen, ${ }^{146}$ S. Brandt,${ }^{146}$ W. Busza, ${ }^{146}$ I. A. Cali, ${ }^{146}$ Z. Demiragli, ${ }^{146}$ L. Di Matteo, ${ }^{146}$ G. Gomez Ceballos, ${ }^{146}$ M. Goncharov ${ }^{146}$ D. Gulhan, ${ }^{146}$ Y. Iiyama, ${ }^{146}$ G. M. Innocenti, ${ }^{146}$ M. Klute, ${ }^{146}$ D. Kovalskyi, ${ }^{146}$ Y. S. Lai, ${ }^{146}$ Y.-J. Lee,${ }^{146}$ A. Levin, ${ }^{146}$ P. D. Luckey, ${ }^{146}$ A. C. Marini, ${ }^{146}$ C. Mcginn, ${ }^{146}$ C. Mironov, ${ }^{146}$ S. Narayanan, ${ }^{146}$ X. Niu, ${ }^{146}$ C. Paus, ${ }^{146}$ C. Roland, ${ }^{146}$ G. Roland, ${ }^{146}$ J. Salfeld-Nebgen, ${ }^{146}$ G. S. F. Stephans,${ }^{146}$ K. Sumorok, ${ }^{146}$ M. Varma, ${ }^{146}$ D. Velicanu, ${ }^{146}$ J. Veverka, ${ }^{146}$ J. Wang, ${ }^{146}$ T. W. Wang, ${ }^{146}$ B. Wyslouch, ${ }^{146}$ M. Yang, ${ }^{146}$ V. Zhukova, ${ }^{146}$ B. Dahmes, ${ }^{147}$ A. Evans, ${ }^{147}$ A. Finkel, ${ }^{147}$ A. Gude, ${ }^{147}$ P. Hansen, ${ }^{147}$ S. Kalafut, ${ }^{147}$ S. C. Kao, ${ }^{147}$ K. Klapoetke, ${ }^{147}$ Y. Kubota, ${ }^{147}$ Z. Lesko, ${ }^{147}$ J. Mans, ${ }^{147}$ S. Nourbakhsh, ${ }^{147}$ N. Ruckstuhl,${ }^{147}$ R. Rusack, ${ }^{147}$ N. Tambe,${ }^{147}$ J. Turkewitz, ${ }^{147}$ J. G. Acosta, ${ }^{148}$ S. Oliveros, ${ }^{148}$ E. Avdeeva, ${ }^{149}$ K. Bloom, ${ }^{149}$ S. Bose, ${ }^{149}$ D. R. Claes, ${ }^{149}$ A. Dominguez, ${ }^{149}$ C. Fangmeier, ${ }^{149}$ R. Gonzalez Suarez, ${ }^{149}$ R. Kamalieddin, ${ }^{149}$ D. Knowlton, ${ }^{149}$ I. Kravchenko, ${ }^{149}$ F. Meier, ${ }^{149}$ J. Monroy, ${ }^{149}$ F. Ratnikov, ${ }^{149}$ J. E. Siado, ${ }^{149}$ G. R. Snow, ${ }^{149}$ M. Alyari, ${ }^{150}$ J. Dolen, ${ }^{150}$ J. George, ${ }^{150}$ A. Godshalk, ${ }^{150}$ C. Harrington, ${ }^{150}$ I. Iashvili, ${ }^{150}$ J. Kaisen, ${ }^{150}$ A. Kharchilava, ${ }^{150}$ A. Kumar, ${ }^{150}$ S. Rappoccio, ${ }^{150}$ B. Roozbahani, ${ }^{150}$ G. Alverson, ${ }^{151}$

E. Barberis, ${ }^{151}$ D. Baumgartel, ${ }^{151}$ M. Chasco, ${ }^{151}$ A. Hortiangtham, ${ }^{151}$ A. Massironi, ${ }^{151}$ D. M. Morse, ${ }^{151}$ D. Nash, ${ }^{151}$ T. Orimoto, ${ }^{151}$ R. Teixeira De Lima, ${ }^{151}$ D. Trocino, ${ }^{151}$ R.-J. Wang, ${ }^{151}$ D. Wood,${ }^{151}$ J. Zhang, ${ }^{151}$ K. A. Hahn, ${ }^{152}$ A. Kubik, ${ }^{152}$ N. Mucia, ${ }^{152}$ N. Odell, ${ }^{152}$ B. Pollack, ${ }^{152}$ M. Schmitt, ${ }^{152}$ S. Stoynev, ${ }^{152}$ K. Sung, ${ }^{152}$ M. Trovato, ${ }^{152}$ M. Velasco, ${ }^{152}$ A. Brinkerhoff, ${ }^{153}$ N. Dev, ${ }^{153}$ M. Hildreth, ${ }^{153}$ C. Jessop, ${ }^{153}$ D. J. Karmgard, ${ }^{153}$ N. Kellams, ${ }^{153}$ K. Lannon, ${ }^{153}$ N. Marinelli, ${ }^{153}$ F. Meng, ${ }^{153}$ C. Mueller, ${ }^{153}$ Y. Musienko, ${ }^{153, \mathrm{~mm}}$ M. Planer, ${ }^{153}$ A. Reinsvold, ${ }^{153}$ R. Ruchti, ${ }^{153}$ G. Smith, ${ }^{153}$ S. Taroni, ${ }^{153}$ N. Valls, ${ }^{153}$ M. Wayne, ${ }^{153}$ M. Wolf,${ }^{153}$ A. Woodard, ${ }^{153}$ L. Antonelli, ${ }^{154}$ J. Brinson, ${ }^{154}$ B. Bylsma, ${ }^{154}$ L. S. Durkin, ${ }^{154}$ S. Flowers, ${ }^{154}$ A. Hart, ${ }^{154}$ C. Hill, ${ }^{154}$ R. Hughes, ${ }^{154}$ W. Ji, ${ }^{154}$ T. Y. Ling, ${ }^{154}$ B. Liu, ${ }^{154}$ W. Luo, ${ }^{154}$ D. Puigh, ${ }^{154}$ M. Rodenburg, ${ }^{154}$ B. L. Winer, ${ }^{154}$ H. W. Wulsin, ${ }^{154}$ O. Driga, ${ }^{155}$ P. Elmer, ${ }^{155}$ J. Hardenbrook, ${ }^{155}$ P. Hebda,${ }^{155}$ S. A. Koay, ${ }^{155}$ P. Lujan ${ }^{155}$ D. Marlow, ${ }^{155}$ T. Medvedeva, ${ }^{155}$ M. Mooney, ${ }^{155}$ J. Olsen, ${ }^{155}$ C. Palmer ${ }^{155}$ P. Piroué ${ }^{155}$ H. Saka, ${ }^{155}$ D. Stickland, ${ }^{155}$ C. Tully, ${ }^{155}$ A. Zuranski, ${ }^{155}$ S. Malik, ${ }^{156}$ V. E. Barnes, ${ }^{157}$ D. Benedetti, ${ }^{157}$ D. Bortoletto,${ }^{157}$ L. Gutay, ${ }^{157}$ M. K. Jha, ${ }^{157}$ M. Jones, ${ }^{157}$ K. Jung, ${ }^{157}$ D. H. Miller, ${ }^{157}$ N. Neumeister, ${ }^{157}$ B. C. Radburn-Smith,${ }^{157}$ X. Shi, ${ }^{157}$ I. Shipsey, ${ }^{157}$ D. Silvers, ${ }^{157}$ J. Sun, ${ }^{157}$ A. Svyatkovskiy, ${ }^{157}$ F. Wang ${ }^{157}$ W. Xie,${ }^{157}$ L. Xu ${ }^{157}$ N. Parashar, ${ }^{158}$ J. Stupak, ${ }^{158}$ A. Adair, ${ }^{159}$ B. Akgun, ${ }^{159}$ Z. Chen ${ }^{159}$ K. M. Ecklund ${ }^{159}$ F. J. M. Geurts, ${ }^{159}$ M. Guilbaud ${ }^{159}$ W. Li,${ }^{159}$ B. Michlin ${ }^{159}$ M. Northup, ${ }^{159}$ B. P. Padley, ${ }^{159}$ R. Redjimi, ${ }^{159}$ J. Roberts, ${ }^{159}$ J. Rorie, ${ }^{159}$ Z. Tu, ${ }^{159}$ J. Zabel,${ }^{159}$ B. Betchart, ${ }^{160}$ A. Bodek,${ }^{160}$ P. de Barbaro, ${ }^{160}$ R. Demina, ${ }^{160}$ Y. Eshaq, ${ }^{160}$ T. Ferbel, ${ }^{160}$ M. Galanti, ${ }^{160}$ A. Garcia-Bellido, ${ }^{160}$ J. Han, ${ }^{160}$ A. Harel, ${ }^{160}$ O. Hindrichs, ${ }^{160}$ A. Khukhunaishvili, ${ }^{160}$ G. Petrillo, ${ }^{160}$ P. Tan, ${ }^{160}$ M. Verzetti, ${ }^{160}$ S. Arora, ${ }^{161}$ A. Barker, ${ }^{161}$ J. P. Chou, ${ }^{161}$

C. Contreras-Campana, ${ }^{161}$ E. Contreras-Campana, ${ }^{161}$ D. Ferencek, ${ }^{161}$ Y. Gershtein, ${ }^{161}$ R. Gray, ${ }^{161}$ E. Halkiadakis, ${ }^{161}$ D. Hidas, ${ }^{161}$ E. Hughes, ${ }^{161}$ S. Kaplan, ${ }^{161}$ R. Kunnawalkam Elayavalli, ${ }^{161}$ A. Lath, ${ }^{161}$ K. Nash, ${ }^{161}$ S. Panwalkar, ${ }^{161}$ M. Park ${ }^{161}$ S. Salur, ${ }^{161}$ S. Schnetzer, ${ }^{161}$ D. Sheffield, ${ }^{161}$ S. Somalwar, ${ }^{161}$ R. Stone, ${ }^{161}$ S. Thomas, ${ }^{161}$ P. Thomassen, ${ }^{161}$ M. Walker, ${ }^{161}$ M. Foerster, ${ }^{162}$ G. Riley, ${ }^{162}$ K. Rose, ${ }^{162}$ S. Spanier ${ }^{162}$ A. York, ${ }^{162}$ O. Bouhali, ${ }^{163, s s s}$

A. Castaneda Hernandez, ${ }^{163, \text { sss }}$ A. Celik, ${ }^{163}$ M. Dalchenko, ${ }^{163}$ M. De Mattia, ${ }^{163}$ A. Delgado, ${ }^{163}$ S. Dildick, ${ }^{163}$ R. Eusebi, ${ }^{163}$ J. Gilmore, ${ }^{163}$ T. Huang, ${ }^{163}$ T. Kamon, ${ }^{163, \text { ttt }}$ V. Krutelyov, ${ }^{163}$ R. Mueller, ${ }^{163}$ I. Osipenkov, ${ }^{163}$ Y. Pakhotin, ${ }^{163}$ R. Patel, ${ }^{163}$ A. Perloff, ${ }^{163}$ A. Rose, ${ }^{163}$ A. Safonov, ${ }^{163}$ A. Tatarinov, ${ }^{163}$ K. A. Ulmer, ${ }^{163, c}$ N. Akchurin, ${ }^{164}$ C. Cowden, ${ }^{164}$ J. Damgov, ${ }^{164}$ C. Dragoiu, ${ }^{164}$ P. R. Dudero, ${ }^{164}$ J. Faulkner, ${ }^{164}$ S. Kunori, ${ }^{164}$ K. Lamichhane, ${ }^{164}$ S. W. Lee, ${ }^{164}$ T. Libeiro, ${ }^{164}$ S. Undleeb, ${ }^{164}$ I. Volobouev, ${ }^{164}$ E. Appelt, ${ }^{165}$ A. G. Delannoy, ${ }^{165}$ S. Greene, ${ }^{165}$ A. Gurrola, ${ }^{165}$ R. Janjam, ${ }^{165}$ W. Johns, ${ }^{165}$ C. Maguire, ${ }^{165}$ Y. Mao, ${ }^{165}$ A. Melo, ${ }^{165}$ H. Ni, ${ }^{165}$ P. Sheldon, ${ }^{165}$ B. Snook, ${ }^{165}$ S. Tuo, ${ }^{165}$ J. Velkovska, ${ }^{165}$ Q. Xu, ${ }^{165}$ M. W. Arenton, ${ }^{166}$ B. Cox, ${ }^{166}$ B. Francis, ${ }^{166}$ J. Goodell, ${ }^{166}$ R. Hirosky, ${ }^{166}$ A. Ledovskoy, ${ }^{166}$ H. Li ${ }^{166}$ C. Lin, ${ }^{166}$ C. Neu, ${ }^{166}$ T. Sinthuprasith, ${ }^{166}$ X. Sun, ${ }^{166}$ Y. Wang, ${ }^{166}$ E. Wolfe, ${ }^{166}$ J. Wood, ${ }^{166}$ F. Xia, ${ }^{166}$ C. Clarke, ${ }^{167}$ R. Harr, ${ }^{167}$ P. E. Karchin, ${ }^{167}$ 
C. Kottachchi Kankanamge Don, ${ }^{167}$ P. Lamichhane,${ }^{167}$ J. Sturdy, ${ }^{167}$ D. A. Belknap, ${ }^{168}$ D. Carlsmith, ${ }^{168}$ M. Cepeda, ${ }^{168}$ S. Dasu, ${ }^{168}$ L. Dodd ${ }^{168}$ S. Duric, ${ }^{168}$ B. Gomber, ${ }^{168}$ M. Grothe, ${ }^{168}$ R. Hall-Wilton, ${ }^{168}$ M. Herndon, ${ }^{168}$ A. Hervé, ${ }^{168}$ P. Klabbers, ${ }^{168}$ A. Lanaro, ${ }^{168}$ A. Levine, ${ }^{168}$ K. Long, ${ }^{168}$ R. Loveless, ${ }^{168}$ A. Mohapatra, ${ }^{168}$ I. Ojalvo, ${ }^{168}$ T. Perry, ${ }^{168}$ G. A. Pierro, ${ }^{168}$ G. Polese, ${ }^{168}$ T. Ruggles, ${ }^{168}$ T. Sarangi, ${ }^{168}$ A. Savin, ${ }^{168}$ A. Sharma, ${ }^{168}$ N. Smith, ${ }^{168}$ W. H. Smith, ${ }^{168}$ D. Taylor, ${ }^{168}$ and N. Woods ${ }^{168}$

(CMS Collaboration)

\author{
${ }^{1}$ Yerevan Physics Institute, Yerevan, Armenia \\ ${ }^{2}$ Institut für Hochenergiephysik der OeAW, Wien, Austria \\ ${ }^{3}$ National Centre for Particle and High Energy Physics, Minsk, Belarus \\ ${ }^{4}$ Universiteit Antwerpen, Antwerpen, Belgium \\ ${ }^{5}$ Vrije Universiteit Brussel, Brussel, Belgium \\ ${ }^{6}$ Université Libre de Bruxelles, Bruxelles, Belgium \\ ${ }^{7}$ Ghent University, Ghent, Belgium \\ ${ }^{8}$ Université Catholique de Louvain, Louvain-la-Neuve, Belgium \\ ${ }^{9}$ Université de Mons, Mons, Belgium \\ ${ }^{10}$ Centro Brasileiro de Pesquisas Fisicas, Rio de Janeiro, Brazil \\ ${ }^{11}$ Universidade do Estado do Rio de Janeiro, Rio de Janeiro, Brazil \\ ${ }^{12 a}$ Universidade Estadual Paulista, São Paulo, Brazil \\ ${ }^{12 \mathrm{~b}}$ Universidade Federal do ABC, São Paulo, Brazil \\ ${ }^{13}$ Institute for Nuclear Research and Nuclear Energy, Sofia, Bulgaria \\ ${ }^{14}$ University of Sofia, Sofia, Bulgaria \\ ${ }^{15}$ Institute of High Energy Physics, Beijing, China \\ ${ }^{16}$ State Key Laboratory of Nuclear Physics and Technology, Peking University, Beijing, China \\ ${ }^{17}$ Universidad de Los Andes, Bogota, Colombia \\ ${ }^{18}$ University of Split, Faculty of Electrical Engineering, Mechanical Engineering and Naval Architecture, Split, Croatia \\ ${ }^{19}$ University of Split, Faculty of Science, Split, Croatia \\ ${ }^{20}$ Institute Rudjer Boskovic, Zagreb, Croatia \\ ${ }^{21}$ University of Cyprus, Nicosia, Cyprus \\ ${ }^{22}$ Charles University, Prague, Czech Republic \\ ${ }^{23}$ Academy of Scientific Research and Technology of the Arab Republic of Egypt, \\ Egyptian Network of High Energy Physics, Cairo, Egypt \\ ${ }^{24}$ National Institute of Chemical Physics and Biophysics, Tallinn, Estonia \\ ${ }^{25}$ Department of Physics, University of Helsinki, Helsinki, Finland \\ ${ }^{26}$ Helsinki Institute of Physics, Helsinki, Finland \\ ${ }^{27}$ Lappeenranta University of Technology, Lappeenranta, Finland \\ ${ }^{28}$ DSM/IRFU, CEA/Saclay, Gif-sur-Yvette, France \\ ${ }^{29}$ Laboratoire Leprince-Ringuet, Ecole Polytechnique, IN2P3-CNRS, Palaiseau, France \\ ${ }^{30}$ Institut Pluridisciplinaire Hubert Curien, Université de Strasbourg, \\ Université de Haute Alsace Mulhouse, CNRS/IN2P3, Strasbourg, France \\ ${ }^{31}$ Centre de Calcul de l'Institut National de Physique Nucleaire et de Physique des Particules, CNRS/IN2P3, Villeurbanne, France \\ ${ }^{32}$ Université de Lyon, Université Claude Bernard Lyon 1, CNRS-IN2P3, Institut de Physique Nucléaire de Lyon, Villeurbanne, France \\ ${ }^{33}$ Georgian Technical University, Tbilisi, Georgia \\ ${ }^{34}$ Tbilisi State University, Tbilisi, Georgia \\ ${ }^{35}$ RWTH Aachen University, I. Physikalisches Institut, Aachen, Germany \\ ${ }^{36}$ RWTH Aachen University, III. Physikalisches Institut A, Aachen, Germany \\ ${ }^{37}$ RWTH Aachen University, III. Physikalisches Institut B, Aachen, Germany \\ ${ }^{38}$ Deutsches Elektronen-Synchrotron, Hamburg, Germany \\ ${ }^{39}$ University of Hamburg, Hamburg, Germany \\ ${ }^{40}$ Institut für Experimentelle Kernphysik, Karlsruhe, Germany \\ ${ }^{41}$ Institute of Nuclear and Particle Physics (INPP), NCSR Demokritos, Aghia Paraskevi, Greece \\ ${ }^{42}$ University of Athens, Athens, Greece \\ ${ }^{43}$ University of Ioánnina, Ioánnina, Greece \\ ${ }^{44}$ Wigner Research Centre for Physics, Budapest, Hungary \\ ${ }^{45}$ Institute of Nuclear Research ATOMKI, Debrecen, Hungary \\ ${ }^{46}$ University of Debrecen, Debrecen, Hungary \\ ${ }^{47}$ National Institute of Science Education and Research, Bhubaneswar, India \\ ${ }^{48}$ Panjab University, Chandigarh, India
}




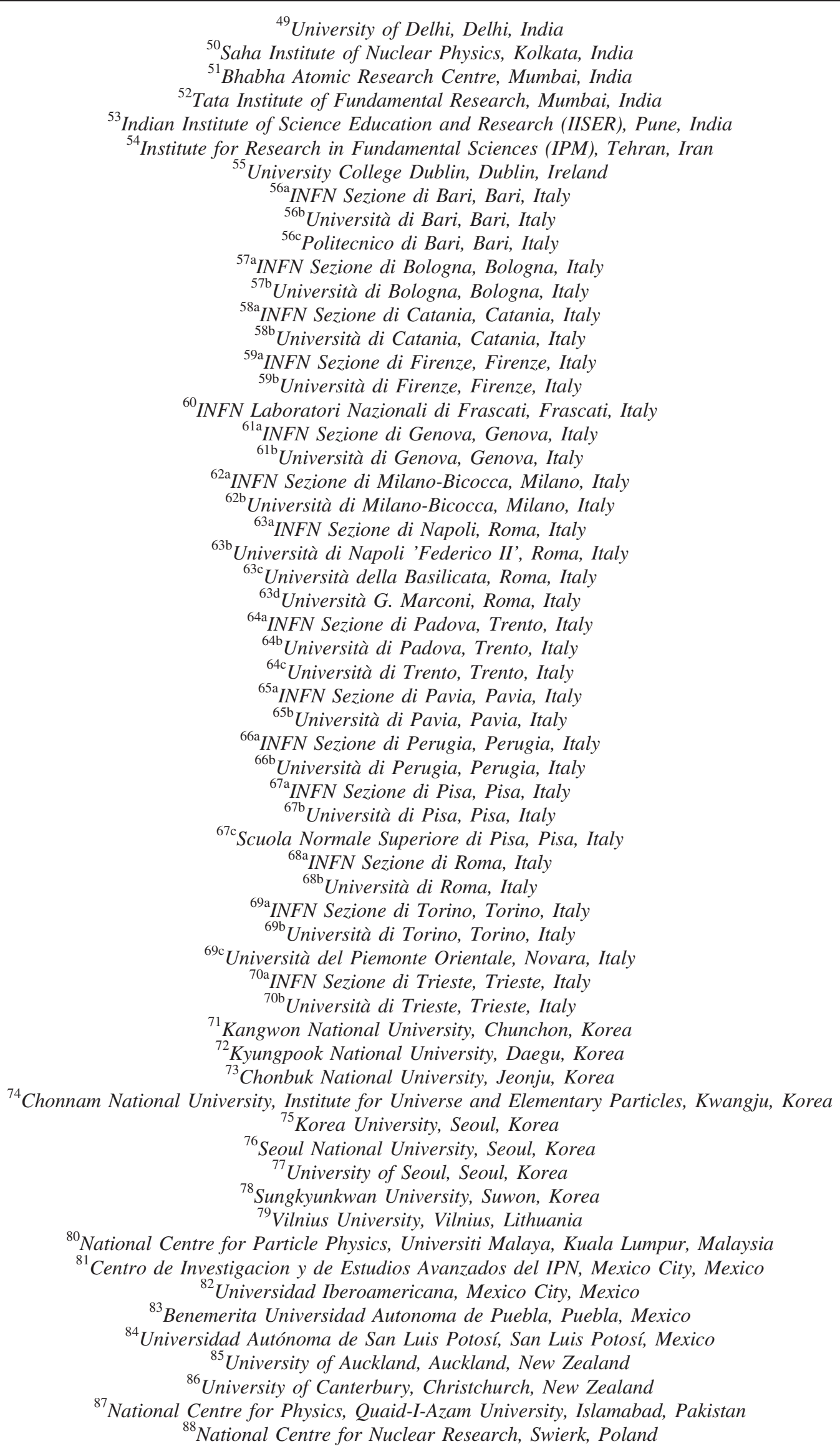


${ }^{89}$ Institute of Experimental Physics, Faculty of Physics, University of Warsaw, Warsaw, Poland

${ }^{90}$ Laboratório de Instrumentação e Física Experimental de Partículas, Lisboa, Portugal

${ }^{91}$ Joint Institute for Nuclear Research, Dubna, Russia

${ }^{92}$ Petersburg Nuclear Physics Institute, Gatchina (St. Petersburg), Russia

${ }^{93}$ Institute for Nuclear Research, Moscow, Russia

${ }^{94}$ Institute for Theoretical and Experimental Physics, Moscow, Russia

${ }^{95}$ National Research Nuclear University 'Moscow Engineering Physics Institute' (MEPhI), Moscow, Russia

${ }^{96}$ P.N. Lebedev Physical Institute, Moscow, Russia

${ }^{97}$ Skobeltsyn Institute of Nuclear Physics, Lomonosov Moscow State University, Moscow, Russia

${ }^{98}$ State Research Center of Russian Federation, Institute for High Energy Physics, Protvino, Russia

${ }^{99}$ University of Belgrade, Faculty of Physics and Vinca Institute of Nuclear Sciences, Belgrade, Serbia

${ }^{100}$ Centro de Investigaciones Energéticas Medioambientales y Tecnológicas (CIEMAT), Madrid, Spain

${ }^{101}$ Universidad Autónoma de Madrid, Madrid, Spain

${ }^{102}$ Universidad de Oviedo, Oviedo, Spain

${ }^{103}$ Instituto de Física de Cantabria (IFCA), CSIC-Universidad de Cantabria, Santander, Spain

${ }^{104}$ CERN, European Organization for Nuclear Research, Geneva, Switzerland

${ }^{105}$ Paul Scherrer Institut, Villigen, Switzerland

${ }^{106}$ Institute for Particle Physics, ETH Zurich, Zurich, Switzerland

${ }^{107}$ Universität Zürich, Zurich, Switzerland

${ }^{108}$ National Central University, Chung-Li, Taiwan

${ }^{109}$ National Taiwan University (NTU), Taipei, Taiwan

${ }^{110}$ Chulalongkorn University, Faculty of Science, Department of Physics, Bangkok, Thailand

${ }^{111}$ Cukurova University, Adana, Turkey

${ }^{112}$ Middle East Technical University, Physics Department, Ankara, Turkey

${ }^{113}$ Bogazici University, Istanbul, Turkey

${ }^{114}$ Istanbul Technical University, Istanbul, Turkey

${ }^{115}$ Institute for Scintillation Materials of National Academy of Science of Ukraine, Kharkov, Ukraine

${ }^{116}$ National Scientific Center, Kharkov Institute of Physics and Technology, Kharkov, Ukraine

${ }^{117}$ University of Bristol, Bristol, United Kingdom

${ }^{118}$ Rutherford Appleton Laboratory, Didcot, United Kingdom

${ }^{119}$ Imperial College, London, United Kingdom

${ }^{120}$ Brunel University, Uxbridge, United Kingdom

${ }^{121}$ Baylor University, Waco, Texas, USA

${ }^{122}$ The University of Alabama, Tuscaloosa, Alabama, USA

${ }^{123}$ Boston University, Boston, Massachusetts, USA

${ }^{124}$ Brown University, Providence, Rhode Island, USA

${ }^{125}$ University of California, Davis, Davis, California, USA

${ }^{126}$ University of California, Los Angeles, Los Angeles, California, USA

${ }^{127}$ University of California, Riverside, Riverside, California, USA

${ }^{128}$ University of California, San Diego, La Jolla, California, USA

${ }^{129}$ University of California, Santa Barbara, Santa Barbara, California, USA

${ }^{130}$ California Institute of Technology, Pasadena, California, USA

${ }^{131}$ Carnegie Mellon University, Pittsburgh, Pennsylvania, USA

${ }^{132}$ University of Colorado Boulder, Boulder, Colorado, USA

${ }^{133}$ Cornell University, Ithaca, New York, USA

${ }^{134}$ Fermi National Accelerator Laboratory, Batavia, Illinois, USA

${ }^{135}$ University of Florida, Gainesville, Florida, USA

${ }^{136}$ Florida International University, Miami, Florida, USA

${ }^{137}$ Florida State University, Tallahassee, Florida, USA

${ }^{138}$ Florida Institute of Technology, Melbourne, Florida, USA

${ }^{139}$ University of Illinois at Chicago (UIC), Chicago, Illinois, USA

${ }^{140}$ The University of Iowa, Iowa City, Iowa, USA

${ }^{141}$ Johns Hopkins University, Baltimore, Maryland, USA

${ }^{142}$ The University of Kansas, Lawrence, Kansas, USA

${ }^{143}$ Kansas State University, Manhattan, Kansas, USA

${ }^{144}$ Lawrence Livermore National Laboratory, Livermore, California, USA

${ }^{145}$ University of Maryland, College Park, Maryland, USA

${ }^{146}$ Massachusetts Institute of Technology, Cambridge, Massachusetts, USA

${ }^{147}$ University of Minnesota, Minneapolis, Minnesota, USA

${ }^{148}$ University of Mississippi, Oxford, Mississippi, USA 


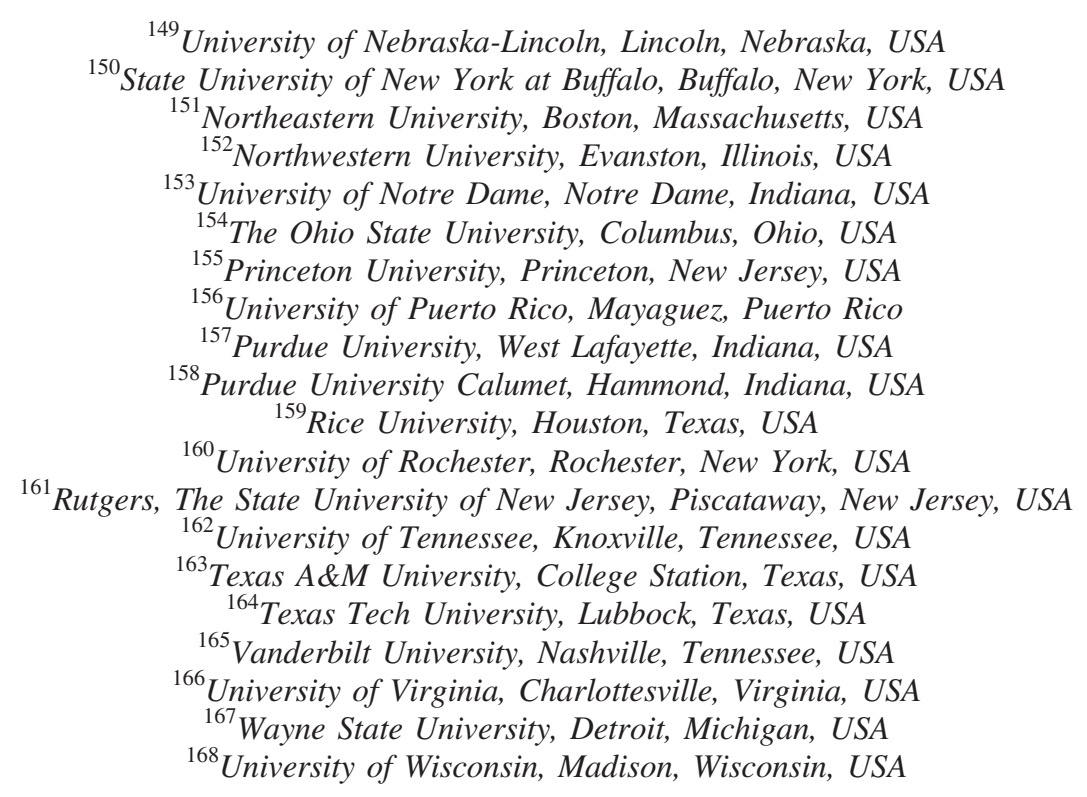

${ }^{\mathrm{a}}$ Deceased.

${ }^{\mathrm{b}}$ Also at Vienna University of Technology, Vienna, Austria.

${ }^{\mathrm{c}}$ Also at CERN, European Organization for Nuclear Research, Geneva, Switzerland.

${ }^{\mathrm{d}}$ Also at State Key Laboratory of Nuclear Physics and Technology, Peking University, Beijing, China.

${ }^{e}$ Also at Institut Pluridisciplinaire Hubert Curien, Université de Strasbourg, Université de Haute Alsace Mulhouse, CNRS/IN2P3, Strasbourg, France.

${ }^{\mathrm{f}}$ Also at National Institute of Chemical Physics and Biophysics, Tallinn, Estonia.

${ }^{\mathrm{g}}$ Also at Skobeltsyn Institute of Nuclear Physics, Lomonosov Moscow State University, Moscow, Russia.

${ }^{\mathrm{h}}$ Also at Universidade Estadual de Campinas, Campinas, Brazil.

${ }^{\mathrm{i}}$ Also at Centre National de la Recherche Scientifique (CNRS) - IN2P3, Paris, France.

${ }^{\mathrm{j}}$ Also at Laboratoire Leprince-Ringuet, Ecole Polytechnique, IN2P3-CNRS, Palaiseau, France.

${ }^{\mathrm{k}}$ Also at Joint Institute for Nuclear Research, Dubna, Russia.

${ }^{1}$ Also at Ain Shams University, Cairo, Egypt.

${ }^{\mathrm{m}}$ Also at Zewail City of Science and Technology, Zewail, Egypt.

${ }^{\mathrm{n}}$ Also at Fayoum University, El-Fayoum, Egypt.

${ }^{\circ}$ Also at British University in Egypt, Cairo, Egypt.

${ }^{\mathrm{p}}$ Also at Université de Haute Alsace, Mulhouse, France.

${ }^{\mathrm{q}}$ Also at Tbilisi State University, Tbilisi, Georgia.

${ }^{\mathrm{r}}$ Also at Ilia State University, Tbilisi, Georgia.

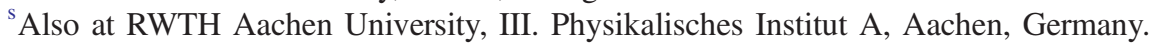

${ }^{\mathrm{t}}$ Also at Indian Institute of Science Education and Research, Bhopal, India.

${ }^{\mathrm{u}}$ Also at University of Hamburg, Hamburg, Germany.

${ }^{v}$ Also at Brandenburg University of Technology, Cottbus, Germany.

${ }^{\mathrm{w}}$ Also at Institute of Nuclear Research ATOMKI, Debrecen, Hungary.

${ }^{\mathrm{x}}$ Also at Eötvös Loránd University, Budapest, Hungary.

${ }^{y}$ Also at University of Debrecen, Debrecen, Hungary.

${ }^{\mathrm{z}}$ Also at Wigner Research Centre for Physics, Budapest, Hungary.

${ }^{\text {aa }}$ Also at University of Visva-Bharati, Santiniketan, India.

${ }^{\mathrm{bb}}$ Also at King Abdulaziz University, Jeddah, Saudi Arabia.

${ }^{\mathrm{cc}}$ Also at University of Ruhuna, Matara, Sri Lanka.

${ }^{\mathrm{dd}}$ Also at Isfahan University of Technology, Isfahan, Iran.

${ }^{\mathrm{ee}}$ Also at University of Tehran, Department of Engineering Science, Tehran, Iran.

${ }^{\mathrm{ff}}$ Also at Plasma Physics Research Center, Science and Research Branch, Islamic Azad University, Tehran, Iran.

${ }^{g g}$ Also at Università degli Studi di Siena, Siena, Italy.

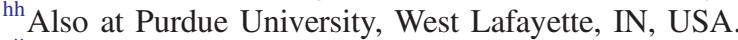

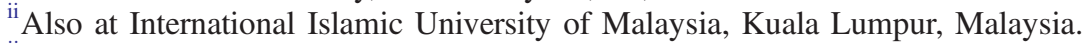

${ }^{\mathrm{jj}}$ Also at Malaysian Nuclear Agency, MOSTI, Kajang, Malaysia.

${ }^{\mathrm{kk}}$ Also at Consejo Nacional de Ciencia y Tecnología, Mexico City, Mexico.

${ }^{11}$ Also at Warsaw University of Technology, Institute of Electronic Systems, Warsaw, Poland. 
${ }^{\mathrm{mm}}$ Also at Institute for Nuclear Research, Moscow, Russia.

${ }^{\mathrm{nn}}$ Also at National Research Nuclear University 'Moscow Engineering Physics Institute' (MEPhI), Moscow, Russia.

${ }^{\text {oo }}$ Also at St. Petersburg State Polytechnical University, St. Petersburg, Russia.

${ }^{\mathrm{pp}}$ Also at California Institute of Technology, Pasadena, CA, USA.

${ }^{\mathrm{qq}}$ Also at Faculty of Physics, University of Belgrade, Belgrade, Serbia.

${ }^{\text {rr }}$ Also at INFN Sezione di Roma, Università di Roma, Roma, Italy.

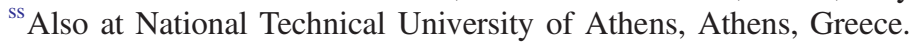

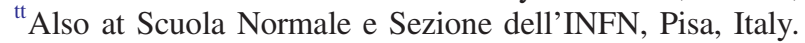

${ }^{\mathrm{uu}}$ Also at University of Athens, Athens, Greece.

${ }^{v v}$ Also at Institute for Theoretical and Experimental Physics, Moscow, Russia.

${ }^{\text {ww }}$ Also at Albert Einstein Center for Fundamental Physics, Bern, Switzerland.

${ }^{\mathrm{xx}}$ Also at Gaziosmanpasa University, Tokat, Turkey.

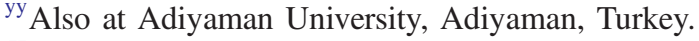

${ }^{\mathrm{zz}}$ Also at Mersin University, Mersin, Turkey.

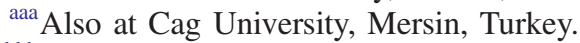

${ }^{\mathrm{bbb}}$ Also at Piri Reis University, Istanbul, Turkey.

${ }^{c c c}$ Also at Ozyegin University, Istanbul, Turkey.

${ }^{\text {ddd }}$ Also at Izmir Institute of Technology, Izmir, Turkey.

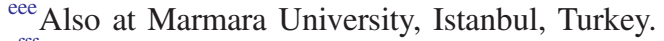

${ }^{\mathrm{fff}}$ Also at Kafkas University, Kars, Turkey.

${ }^{\text {ggg }}$ Also at Istanbul Bilgi University, Istanbul, Turkey.

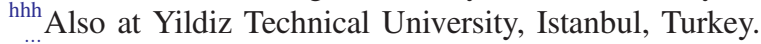

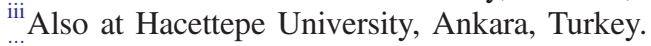

${ }^{\mathrm{jjj}}$ Also at Rutherford Appleton Laboratory, Didcot, United Kingdom.

${ }^{k k k}$ Also at School of Physics and Astronomy, University of Southampton, Southampton, United Kingdom.

${ }^{111}$ Also at Instituto de Astrofísica de Canarias, La Laguna, Spain.

${ }^{\mathrm{mmm}}$ Also at Utah Valley University, Orem, UT, USA.

${ }^{n n n}$ Also at University of Belgrade, Faculty of Physics and Vinca Institute of Nuclear Sciences, Belgrade, Serbia.

${ }^{\circ 00}$ Also at Facoltà Ingegneria, Università di Roma, Roma, Italy.

${ }^{\mathrm{ppp}}$ Also at Argonne National Laboratory, Argonne, IL, USA.

${ }^{\mathrm{qqq}}$ Also at Erzincan University, Erzincan, Turkey.

${ }^{\mathrm{rrr}}$ Also at Mimar Sinan University, Istanbul, Istanbul, Turkey.

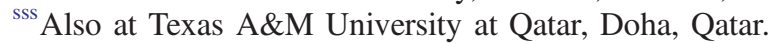

${ }^{\text {ttt }}$ Also at Kyungpook National University, Daegu, Korea. 